\title{
Nonextendible Positive Maps
}

\author{
S. L. Woronowicz * \\ ZiF, University of Bielefeld, D-4800 Bielefeld, Federal Republic of Germany
}

\begin{abstract}
Positive maps of ordered vector spaces into the algebra of all bounded operators acting on a Hilbert space are considered. A special class of so called nonextendible maps is introduced and investigated. This class is much smaller than the class of extreme maps.

Any positive map can be obtained from a nonextendible one by restriction.

In the $C^{*}$-algebra case, the nonextendibility of a normalized positive map $\phi$ is related to the properties of the expression $\phi\left(a^{2}\right)-\phi(a)^{2}$. In particular Jordan representations are non-extendible.

2-positive nonextendible maps are representations. Similar result holds for copositive maps. For abelian $C^{*}$-algebras, notion of nonextendible map and that of representation coincide.

The nonextendible positive maps of the Jordan algebra $M_{2 s}$ of all $2 \times 2$ symmetric matrices and of the full $2 \times 2$ matrix algebra are especially investigated. Any nonextendible normalized positive map of $M_{2 s}$ is a Jordan representation. $M_{2}$ admits nonextendible normalized positive maps not being Jordan representations. A large class of examples is given.
\end{abstract}

\section{Introduction}

Let $\mathfrak{A}$ and $\mathfrak{B}$ be $C^{*}$-algebras. We denote by $\mathfrak{A}_{+}$and $\mathfrak{B}_{+}$the cones of positive elements and by $1_{\mathfrak{Q}}$ and $1_{\mathfrak{B}}$ the unity elements of these algebras. We shall consider linear maps $\phi$ of $\mathfrak{A}$ into $\mathfrak{B}$ such that $\phi\left(\mathfrak{U}_{+}\right) \subset \mathfrak{B}_{+}$and $\phi\left(1_{\mathfrak{r}}\right)=1_{\mathfrak{B}}$. To stress these properties we write

$$
\phi:\left(\mathfrak{A}, \mathfrak{A}_{+}, 1_{\mathfrak{U}}\right) \rightarrow\left(\mathfrak{B}, \mathfrak{B}_{+}, 1_{\mathfrak{B}}\right) .
$$

We say that $\phi$ is a normalized positive map. In recent years, positive maps have become of common interest to mathematicians and physicists in particular in connection with the operator theory (cf. [1]) and the quantum theory of open systems (cf. $[6,8,12])$. The notion of positive map generalizes that of state, re-

* On leave of absence from: Department of Mathematical Methods in Physics, University of Warsaw, Hoża 74, 00-682 Warsaw, Poland 
presentation, Jordan representation, conditional expectation and (semi-) spectral measure. As it is shown in the beautiful review article [18] of Størmer, many properties and constructions related to these particular concepts can be generalized to larger classes of positive maps giving rise to the general theory. We refer to this article for basic definitions and results.

Despite the great interest, the existing theory of positive maps contains no convenient formula expressing all positive maps in terms of a smaller class of objects having a richer structure and exhibiting interesting properties.

In this paper we present a new approach to this problem. This approach is suggested by the Stinespring theorem.

Let us recall that $(0.1)$ is called $n$-positive (where $n$ is an integer number) if the tensor product map

$$
\phi \otimes i d: \mathfrak{A} \otimes M_{n} \rightarrow \mathfrak{B} \otimes M_{n}
$$

is positive. Here $M_{n}$ denotes the algebra of all $n \times n$ matrices with complex entries. A map is called completely positive if it is $n$-positive for every $n$.

Let $H$ be a Hilbert space and $B(H)$ be the algebra of all bounded operators acting on $H$. The Stinespring theorem says that

$$
\phi: \mathfrak{U} \rightarrow B(H)
$$

is a normalized completely positive map iff

$$
\phi(a)=P \tilde{\phi}(a) P
$$

where $\tilde{\phi}$ is a representation of $\mathfrak{U}$ acting on a Hilbert space $\tilde{H}$ containing $H$ and $P: \tilde{H} \rightarrow H$ is the orthogonal projection. We refer to the formula $(0.4)$ saying that $\tilde{\phi}$ is an extension of $\phi$.

One may introduce the notion of copositive map replacing in $(0.2)$ the identity map by the transposition of $n \times n$-matrices [20]. It turns out that $(0.3)$ is a completely copositive normalized map iff it can be extended to a corepresentation.

We say that a map $\phi$ is of jordanian type if it splits into sum of completely positive and completely copositive maps. In virtue of the foregoing and a result of Kadison [10]: (0.3) is of jordanian type iff it can be extended to a Jordan representation (this fact justifies our terminology).

Only for a few pairs of $C^{*}$-algebras $(\mathfrak{A}, \mathfrak{B})$ all positive maps from $\mathfrak{A}$ into $\mathfrak{B}$ are of jordanian type. As far as matrix algebras are concerned, this is the case only for $\left(M_{1}, M_{n}\right),\left(M_{2}, M_{2}\right),\left(M_{2}, M_{3}\right),\left(M_{3}, M_{2}\right)$ and $\left(M_{n}, M_{1}\right)$ (cf. [20]).

We would like to point out that, although positive maps of non-jordanian type are widerspread (as it is seen from the above list), the first concrete example of such a map was found only recently [4]. This discovery was unexpected. It turned out that the formula (0.4) does not exhaust all normalized positive maps when $\tilde{\phi}$ runs over all Jordan representations of $\mathfrak{A}$.

In our theory we replace the class of Jordan representations by a larger class of positive maps; the members of this larger class are called nonextendible positive maps. The precise definition of this notion will be given in the next section. Intuitively, a map $\phi$ is nonextendible if it can not be written in the form (0.4), where $\tilde{\phi}$ is another positive map, in a nontrivial way.

It turns out that any normalized positive map can be extended to a nonextendible one. Therefore the Formula (0.4) expresses all normalized positive 
maps (0.3) in terms of nonextendible ones. We shall see that the nonextendibility of a map implies many interesting properties. For example any normalized nonextendible map is extreme in the cone of all positive maps.

This fact shows the essential advantage of our approach over the program based on the Krein-Milman-Choquet theory [16]. By using the standard methods of the theory of compact convex sets one gets a formula expressing all normalized positive maps in terms of extreme ones. However the extreme positive maps do not exhibit interesting properties and do not admit any good description (cf. [4]). Moreover if $\mathfrak{Q}$ is not separable, then we meet difficulties related to the measure theory.

In our theory we deal only with a small special subclass of extreme positive maps and the problem of good description is not so hopeless as in the case when all extreme positive maps are involved.

Let us shortly describe the contents of the paper. The three first sections are devoted to the theory of positive maps of ordered vector spaces into $B(H)$. There are many reasons for this generalization. Ordered vector spaces form a natural domain for positive maps. We hope that the detail investigation of the set of all positive maps defined on such a space will result in a better understanding of the structure of the space.

In the first section we introduce the basic concepts and state the main results of the extension theory. The Section 2 proves that any normalized positive map admits a nonextendible extension.

On the way we derive interesting conditions characterizing nonextendible maps. These conditions are expressed in terms of a seminorm associated with the investigated positive map.

The definition of the seminorm given in the Section 2 is not convenient for applications. In the Section 3 we introduce the seminorm in another equivalent way, not related directly to the extension theory. As a result we get an interesting sufficient condition for the nonextendibility in the finite-dimensional case. We use this condition in the Section 6.

The Section 4 is devoted to the study of nonextendible positive maps of $C^{*}$-algebras. We discover a remarkable relation between the Kadison inequality and extension properties. In particular Jordan representations turn out to be nonextendible. We prove that nonextendible 2-positive maps are representations. The similar result holds for 2-copositive maps. Consequently the representations are the only nonextendible normalized positive maps of abelian $C^{*}$-algebras.

In the Section 5 we investigate the nonextendible positive maps of the Jordan algebra of all symmetric $2 \times 2$-matrices with complex entries. It turns out that any such a map is a Jordan representation. Then, applying our main theorem we obtain many facts about the structure of any positive map of this algebra. Some of these facts were known before (cf. [2, 3,9], [11], Lemma 4, p. 15, [17]).

In the Section 6 we deal with the full algebra of $2 \times 2$-matrices with complex entries. It turns out that in this case there exist nonextendible normalized positive maps not being Jordan representations. A large class of examples is presented. We hope that the further investigations with the use of the methods elaborated in this section will lead to a good description of the set of all nonextendible maps of $M_{2}$. 


\section{Ordered Vector Spaces and Positive Maps}

One can easily notice that the notion of a normalized positive map is not related to the whole $C^{*}$-algebra structure. Indeed, only the linear structure of $\mathfrak{A}$, the cone $\mathfrak{A}_{+}$and the distinguished element $1_{\mathscr{N}}$ are involved in the definition given in the beginning of this paper. The most general structure dealing with these formations is that of an ordered vector space with the unity. The following definition copies the well known properties of $C^{*}$-algebras.

Definition 1.1. Let $\mathfrak{A}$ be a complex vector space, $\mathfrak{A}_{+}$be a cone in $\mathfrak{A}$ (i.e. a convex subset such that $\lambda \mathfrak{A}_{+} \subset \mathfrak{A}_{+}$for any $\lambda \geqq 0$ ) and 1 be an element of $\mathfrak{A}_{+}$. We say that $\left(\mathfrak{U}, \mathfrak{U}_{+}, 1\right)$ is an ordered vector space with the unity if

1. Any element $a \in \mathfrak{A}$ admits the unique decomposition

$a=a_{1}+i a_{2}$

where $a_{1}, a_{2} \in \mathfrak{A}_{r}$ and $\mathfrak{A}_{r}$ denotes the real vector subspace generated by $\mathfrak{A}_{+}: \mathfrak{A}_{r}=$ $\mathfrak{U}_{+}-\mathfrak{U}_{+}$.

2. For any $a \in \mathfrak{A}_{+}$one can find $\lambda \in \mathbb{R}$ such that

$\lambda 1-a \in \mathfrak{A}_{+}$.

3. $\mathfrak{A}_{+} \cap\left(-\mathfrak{A}_{+}\right)=\{0\}$.

The word "ordered" refers to the following partially ordering relation $\geqq$ compatible with the vector space structure:

$(a \geqq b) \quad$ iff $\quad\left(a-b \in \mathfrak{A}_{+}\right)$

for any $a, b \in \mathfrak{A}$. Elements of $\mathfrak{A}_{r}$ are called selfadjoint or hermitian. They are characterized by the equation $a^{*}=a$, where $*$-operation is defined in the usual way: if $a$ is given by (1.1) then $a^{*}=a_{1}-i a_{2}$.

Although the structure described in Definition 1.1 is very simple, the theory of ordered vector spaces contains many nontrivial results. One problem is specially interesting: What are conditions distinguishing $C^{*}$-algebras and Jordan algebras among other ordered vector spaces with the unity. This question is of great importance especially with its relation to the modern approach to the foundations of quantum mechanics [15]. We believe that the existence of the complete system of selfpolar forms [19] besides some obvious topological conditions is characteristic for Jordan algebras. It seems that the most natural approach to this problem consists in an investigation of positive maps of ordered vector spaces into $B(H)$.

It is obvious, what is meant by a normalized positive map of ordered vector spaces with the unity. We give the formal definition for the case when the target space is the algebra of all bounded operators acting on a Hilbert space (this is the only case, we are interested in this paper).

Definition 1.2. Let $\left(\mathfrak{U}, \mathfrak{U}_{+}, 1\right)$ be an ordered vector space with the unity and

$$
\phi: \mathfrak{A} \rightarrow B(H)
$$

be a linear map. We say that $\phi$ is a normalized positive map if $\phi(a) \geqq 0$ for any $a \in \mathfrak{U}_{+}$and $\phi(1)=I$. In this case we write

$$
\phi:\left(\mathfrak{A}, \mathfrak{A}_{+}, 1\right) \rightarrow\left(B(H), B_{+}(H), I\right)
$$


where obviously $B_{+}(H)$ denotes the set of all positive operators and $I$ is the unity of $B(H)$.

The Hilbert space $H$ is called the carrier Hilbert space of $\phi$.

Let $\left(\mathfrak{U}, \mathfrak{U}_{+}, 1\right)$ be an ordered vector space with the unity and $a \in \mathfrak{A}_{r}$. It follows easily from (1.2) that for sufficiently large $\lambda$

$$
-\lambda 1 \leqq a \leqq \lambda 1 \text {. }
$$

We denote by $\|a\|$ the greatest lower bound of the set of $\lambda$ 's satisfying (1.4). The function $\|\cdot\|$ is a seminorm on $\mathfrak{A}_{r}$ [to prove that it is a norm, one needs a little stronger version of (1.3)]. All topological properties of $\mathfrak{U}_{r}$ will be refered to the topology defined by this seminorm.

Let us notice that 1 is an interior point of $\mathfrak{U}_{+} \subset \mathfrak{U}_{r}$. Indeed, if $a \in \mathfrak{U}_{r}$ and $\|a-1\| \leqq \frac{1}{2}$ then $-1 \leqq a-1$ and $0 \leqq a$ i.e. $a \in \mathfrak{A}_{+}$. This fact is very important. To get any reasonable Hahn-Banach type theorem for positive functionals one has to assume that the cone has interior points.

It can be easily shown that normalized positive maps are continuous. The very reason is that the topological structure is determined by the ordering.

Definition 1.3. Let $\left(\mathfrak{U}, \mathfrak{U}_{+}, 1\right)$ be an ordered vector space with the unity and

$$
\begin{aligned}
& \phi:\left(\mathfrak{U}, \mathfrak{U}_{+}, 1\right) \rightarrow\left(B(H), B_{+}(H), I_{H}\right), \\
& \tilde{\phi}:\left(\mathfrak{U}, \mathfrak{A}_{+}, 1\right) \rightarrow\left(B(\tilde{H}), B_{+}(\tilde{H}), I_{\tilde{H}}\right) .
\end{aligned}
$$

We say that $\tilde{\phi}$ is an extension of $\phi(\phi$ is a restriction of $\tilde{\phi})$ and write $\tilde{\phi} \supset \phi$ if $\tilde{H} \supset H$ and

$$
\phi(a)=P \tilde{\phi}(a) P
$$

for any $a \in \mathfrak{A} ; P$ in the above formula denotes the orthogonal projection $\tilde{H}$ onto $H$. The extension $\tilde{\phi} \supset \phi$ is called elementary (resp. finite) if $\operatorname{dim} \tilde{H} / H \leqq 1$ (resp. $\operatorname{dim} \tilde{H} / H<\infty)$.

Let $\phi$ and $\phi_{1}$ be two normalized positive maps of $\left(\mathfrak{A}, \mathfrak{U}_{+}, 1\right)$ with the carrier Hilbert spaces $H$ and $H_{1}$ respectively. One can easily introduce the direct sum $\left(\phi \oplus \phi_{1}\right)(a)=\phi(a) \oplus \phi_{1}(a)$. This is a positive map of $\left(\mathfrak{H}, \mathfrak{A}_{+}, 1\right)$ with the carrier Hilbert space $H \oplus H_{1}$. Clearly $\phi \oplus \phi_{1}$ is an extension of $\phi$. We call such extensions trivial.

Definition 1.4. An extension $\tilde{\phi} \supset \phi$ is called trivial if the carrier Hilbert space $H$ of the map $\phi$ is invariant under the action of operators $\tilde{\phi}(a)$ for all $a \in \mathfrak{U}$. Then

$$
\tilde{\phi}(a) h=\phi(a) h
$$

for all $a \in \mathfrak{A}$ and $h \in H$.

Remark. In many cases the inclusion $H \subset \tilde{H}$ mentioned in Definition 1.3 is realized by an isometric embeding

$$
i: H \rightarrow \tilde{H} \text {. }
$$

Then the formulae (1.5) and (1.6) should be replaced by

$$
\phi(a)=i^{*} \tilde{\phi}(a) i
$$


and

$$
\tilde{\phi}(a) i(h)=i(\phi(a) h)
$$

respectively.

Let $\phi$ be a normalized positive map of $\left(\mathfrak{A}, \mathfrak{A}_{+}, 1\right), H$ be the carrier Hilbert space. of $\phi, a \in \mathfrak{A}$ and $h \in H$. Sometimes it is more convenient to write $\phi(a \otimes h)$ instead of $\phi(a) h$. By linearity one may define $\phi(\alpha)$ for any $\alpha \in \mathfrak{A} \otimes H$ :

$$
\phi\left(\sum a_{i} \otimes h_{i}\right)=\sum \phi\left(a_{i}\right) h_{i}
$$

Clearly $\phi(\alpha) \in H$.

If $\tilde{\phi} \supset \phi$ and $\alpha \in \mathfrak{U} \otimes H$ then $\tilde{\phi}(\alpha)$ and $\phi(\alpha)$ are well defined and $\phi(\alpha)=P \tilde{\phi}(\alpha)$. Therefore

$$
\|\phi(\alpha)\| \leqq\|\tilde{\phi}(\alpha)\| .
$$

If the extension $\tilde{\phi} \supset \phi$ is trivial then $\tilde{\phi}(\alpha) \in H$ and we get the equality in (1.10). Conversely if for any $\alpha \in \mathfrak{A} \otimes H$

$$
\|\phi(\alpha)\|=\|\tilde{\phi}(\alpha)\|
$$

then $\tilde{\phi}(\alpha)=\phi(\alpha)$ and the extension $\tilde{\phi} \supset \phi$ is trivial. It means that the property (1.11) is characteristic for trivial extensions.

Now, we introduce the basic notion of this paper

Definition 1.5. Let $\left(\mathfrak{U}, \mathfrak{U}_{+}, 1\right)$ be an ordered vector space with the unity and

$$
\phi:\left(\mathfrak{U}, \mathfrak{U}_{+}, 1\right) \rightarrow\left(B(H), B_{+}(H), I\right) .
$$

We say that $\phi$ is a nonextendible normalized positive map if it admits only trivial extensions. In other words $\phi$ is nonextendible if

$$
\|\phi(\alpha)\|=\|\tilde{\phi}(\alpha)\|
$$

for any $\tilde{\phi} \supset \phi$ and $\alpha \in \mathfrak{U} \otimes H$.

In the next sections we find many interesting properties of nonextendible maps. Here we show that the nonextendibility is the more restrictive property than the extremality (in the sense of the theory of convex sets [16]).

Theorem 1.6. Nonextendible normalized positive maps are extreme in the convex set of all normalized positive maps. Irreducible nonextendible positive maps belong to the extreme rays of the convex cone of all positive maps $\left(\mathfrak{A}, \mathfrak{U}_{+}\right) \rightarrow\left(B(H), B_{+}(H)\right)$.

Let us remind that a map $\phi: \mathfrak{U} \rightarrow B(H)$ is irreducible if the scalars (multiples of $I$ ) are the only operators commuting with all $\phi(a)$.

Proof. Let

$$
\phi:\left(\mathfrak{U}, \mathfrak{U}_{+}, 1\right) \rightarrow\left(B(H), B_{+}(H), I\right)
$$

be nonextendible. Assume that

$$
\phi=\lambda \phi_{1}+\mu \phi_{2}
$$

where $\phi_{1}, \phi_{2}$ are linear maps $\left(\mathfrak{U}, \mathfrak{U}_{+}\right) \rightarrow\left(B(H), B_{+}(H)\right), \lambda, \mu$ are positive numbers and $\lambda+\mu=1$. 
Let $H_{1}$ and $H_{2}$ denote the closure of $\phi_{1}(1) H$ and $\phi_{2}(1) H$ respectively. Then $\phi_{1}(1)$ is invertible on $H_{1}$ we denote by $\phi_{1 n}$ the normalization of $\phi_{1}$ :

$$
\phi_{1 n}(a)=\phi_{1}(1)^{-1 / 2} \phi_{1}(a) \phi_{1}(1)^{-1 / 2} .
$$

One can easily show that this formula defines a bounded operator acting on $H_{1}$. In the same way we introduce $\phi_{2 n}$ :

$$
\phi_{2 n}(a)=\phi_{2}(1)^{-1 / 2} \phi_{2}(a) \phi_{2}(1)^{-1 / 2} \text {. }
$$

Let

$$
\begin{aligned}
& \tilde{H}=H_{1} \oplus H_{2} \\
& \tilde{\phi}=\phi_{1 n} \oplus \phi_{2 n} .
\end{aligned}
$$

Then

$$
\tilde{\phi}:\left(\mathfrak{A}, \mathfrak{U}_{+}, 1\right) \rightarrow\left(B(\tilde{H}), B_{+}(\tilde{H}), I_{\tilde{H}}\right) .
$$

Let

$$
i: H \rightarrow \tilde{H}
$$

be a linear map introduced by

$$
i(h)=\left(\lambda \phi_{1}(1)\right)^{1 / 2} h \oplus\left(\mu \phi_{2}(1)\right)^{1 / 2} h .
$$

Using (1.13)-(1.16) and (1.18) we get immediately

$$
(k \mid \phi(a) h)=(i(k) \mid \tilde{\phi}(a) i(h))
$$

for any $k, h \in H$ and $a \in \mathfrak{A}$. Setting here $a=1$ we prove that (1.17) is an isometric embeding. Now, the above formula means that $\tilde{\phi}$ is an extension of $\phi$ (cf. (1.7)). We assumed that $\phi$ is nonextendible. Thus $\tilde{\phi} \supset \phi$ is a trivial extension. In virtue of (1.8), for any $a \in \mathfrak{U}$ and $h \in H$ we have

$$
\tilde{\phi}(a) i(h)=i(\phi(a) h) \text {. }
$$

Using (1.16) and (1.18) one may rewrite this formula in the more explicite way:

$$
\begin{aligned}
& \lambda^{1 / 2} \phi_{1}(1)^{-1 / 2} \phi_{1}(a) h \oplus \mu^{1 / 2} \phi_{2}(1)^{-1 / 2} \phi_{2}(a) h \\
& =\lambda^{1 / 2} \phi_{1}(1)^{1 / 2} \phi(a) h \oplus \mu^{1 / 2} \phi_{2}(1)^{1 / 2} \phi(a) h .
\end{aligned}
$$

It shows that

$$
\phi_{1}(a)=\phi_{1}(1) \phi(a)
$$

and

$$
\phi_{2}(a)=\phi_{2}(1) \phi(a) .
$$

Assume now that $\phi_{1}$ and $\phi_{2}$ are normalized. Then we get $\phi_{1}(a)=\phi_{2}(a)=\phi(a)$ and the first part of the theorem is proved.

To prove the second part we remind that the product of two hermitian operators is hermitian only if the operators commute. Therefore the formula (1.19) implies that $\phi_{1}(1)$ commute with all $\phi(a)$. If $\phi$ is irreducible then $\phi_{1}(1)$ is a scalar operator and $\phi_{1}$ is proportional to $\phi$. The same holds for $\phi_{2}$. Q.E.D. 
The following theorem shows that the classification of normalized positive maps can be reduced to that of nonextendible maps. This is the main result of the paper, as far as the general setting is concerned.

Theorem 1.7. Any normalized positive map $\phi$ of $\left(\mathfrak{U}, \mathfrak{U}_{+}, 1\right)$ admits a nonextendible extension $\tilde{\phi} \supset \phi$. If $\mathfrak{A}$ and the carrier Hilbert space of $\phi$ are separable, then one may choose $\tilde{\phi}$ such that its carrier Hilbert space is seperable.

The proof of this theorem will be given in the next section. As one may expect, it uses the methods related to the Zorn's lemma.

\section{Extension Theory for Positive Maps}

This section is mainly devoted to the proof of Theorem 1.7. We show that the process of successive extensions can be carried out in such a way that in the end it produces a nonextendible map. On the way we get interesting properties characteristic for nonextendible positive maps.

Let $\left(\mathfrak{U}, \mathfrak{U}_{+}, 1\right)$ be an ordered vector space with the unity, $H$ be a Hilbert space and

$$
\phi:\left(\mathfrak{U}, \mathfrak{U}_{+}, 1\right) \rightarrow\left(B(H), B_{+}(H), I\right) .
$$

We shall consider the set of all elementary extensions of $\phi$. It will be denoted by $\mathrm{el}(\phi)$. One may assume that the carrier Hilbert space of these extensions coincides with $H_{1}=H \oplus \mathbb{C}$. In el $(\phi)$ we introduce the topology induced by the weak operator topology of $B\left(H_{1}\right)$. Remembering that weakly closed bounded subsets of $B\left(H_{1}\right)$ are weakly compact, one can easily show that el $(\phi)$ is compact.

Let us fix an element $\alpha \in \mathfrak{A} \otimes H$. We shall use the notation introduced by (1.9). Denoting by $y \in H_{1}$ a normalized vector orthogonal to $H$ we have $\left\|\phi_{1}(\alpha)\right\|^{2}=$ $\|\phi(\alpha)\|^{2}+\left|\left(y \mid \phi_{1}(\alpha)\right)\right|^{2}$ for any $\phi_{1} \in \operatorname{el}(\phi)$. It follows easily that

$$
\mathrm{el}(\phi) \ni \phi_{1} \rightarrow\left\|\phi_{1}(\alpha)\right\|
$$

is a continuous function. Any continuous function defined on a compact set attains its maximum value. The maximum value of (2.2) will be denoted by $\|\alpha\|_{\phi}$ :

$$
\|\alpha\|_{\phi}=\max _{\phi_{1} \in \mathrm{el}(\phi)}\left\|\phi_{1}(\alpha)\right\| \text {. }
$$

Clearly (2.3) introduces a seminorm on $\mathfrak{U} \otimes H$. As we shall see later, this seminorm plays an essential role in the extension theory. The Definition (2.3) is adapted to the main purpose of this section i.e. to the proof of Theorem 1.7 and is not convenient for other applications. In the next section we introduce this seminorm in another way without any reference to extensions of $\phi$.

Let us note that (2.3) implies the similar formula involving all (not necessarily elementary) extensions of $\phi$ :

$$
\|\alpha\|_{\phi}=\sup _{\tilde{\phi} \supset \phi}\|\tilde{\phi}(\alpha)\| \text {. }
$$

Indeed, given an extension $\tilde{\phi} \supset \phi$ and an element $\alpha \in \mathfrak{A} \otimes H$, one may consider the restriction (in the sense of Def. 1.3) of $\tilde{\phi}$ to the subspace spanned by $H$ and $\tilde{\phi}(\alpha)$. 
Denoting this restriction by $\phi_{1}$ we have $\phi_{1} \in \operatorname{el}(\phi)$ and $\tilde{\phi}(\alpha)=\phi_{1}(\alpha)$. Therefore $\|\tilde{\phi}(\alpha)\|=\left\|\phi_{1}(\alpha)\right\| \leqq\|\alpha\|_{\phi}$ and (2.4) follows.

For some elements $\alpha \in \mathfrak{U} \otimes H$ the seminorm $\|\alpha\|_{\phi}$ can be easily calculated. Let $\alpha=1 \otimes h$, where $h \in H$. Since $\tilde{\phi}(1)=I$ for any extension $\tilde{\phi} \supset \phi, \tilde{\phi}(\alpha)=h$ and $(2.4)$ implies immediately

$$
\|1 \otimes h\|_{\phi}=\|h\| \text {. }
$$

We shall give another application of (2.4).

Lemma 2.1. Let $\phi \subset \tilde{\phi}$ and $\alpha \in \mathfrak{U} \otimes H$ (where $H$ is the carrier Hilbert space of $\phi)$. Then

$$
\|\alpha\|_{\phi} \geqq\|\alpha\|_{\tilde{\phi}} \geqq\|\tilde{\phi}(\alpha)\| \geqq\|\phi(\alpha)\| .
$$

In particular

$$
\left(\|\alpha\|_{\phi}=\|\phi(\alpha)\|\right) \Rightarrow\left(\|\alpha\|_{\tilde{\phi}}=\|\tilde{\phi}(\alpha)\|\right) .
$$

Proof. The left inequality follows from (2.4), because any extension of $\tilde{\phi}$ is an extension of $\phi$. The middle inequality also follows from (2.4): $\tilde{\phi}$ is an extension of $\tilde{\phi}$. The last part of (2.6) coincides with (1.10).

Q.E.D.

The last statement of the Lemma 2.1 is very important. It says that the relation

$$
\|\alpha\|_{\phi}=\|\phi(\alpha)\|
$$

can not be destroyed by passing to an extension. The next proposition shows that this relation plays a crucial role in the theory of nonextendible maps. It gives the nice characterization of these maps.

Proposition 2.2. Let $\phi:\left(\mathfrak{A}, \mathfrak{A}_{+}, 1\right) \rightarrow\left(B(H), B_{+}(H), I\right)$. Then the following conditions are equivalent :

(a) $\phi$ is nonextendible.

(b) For any $\alpha \in \mathfrak{U} \otimes H$ :

$$
\|\alpha\|_{\phi}=\|\phi(\alpha)\| \text {. }
$$

(c) There exist total subsets $\mathfrak{H}_{0} \subset \mathfrak{A}$ and $H_{0} \subset H$ such that

$$
\|a \otimes h\|_{\phi}=\|\phi(a) h\|
$$

for any $a \in \mathfrak{U}_{0}$ and $h \in H_{0}$.

Let us recall that a subset of a topological vector space is said to be total if it generates a dense subspace.

(d) Any linear functional $f$ on $\mathfrak{U} \otimes H$ continuous with respect to $\|\cdot\|_{\phi}$ is of the form

$$
f(a \otimes h)=(y \mid \phi(a) h)
$$

where $y$ is a fixed vector belonging to $H$.

(e) Let $N_{\phi}=\left\{\alpha \in \mathfrak{U} \otimes H:\|\alpha\|_{\phi}=0\right\}$. Then $N_{\phi}$ contains the kernel of the mapping $\mathfrak{U} \otimes H \ni \alpha \rightarrow \phi(\alpha) \in H$. 
Proof. The scheme of the proof:

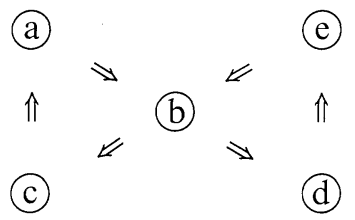

(a) $\Rightarrow$ (b). Assume that $\phi$ is nonextendible. Then (cf. Def. 1.5) $\tilde{\phi}(\alpha)=\phi(\alpha)$ for any $\phi \supset \phi .(2.7)$ is now implied by (2.4).

(b) $\Rightarrow$ (c). It is obvious.

(c) $\Rightarrow$ (a). Assume (2.8). Let $\tilde{\phi}$ be an extension of $\phi$. Then, in virtue of (2.4) $\|\tilde{\phi}(a) h\| \leqq\|\phi(a) h\|$.

On the other hand $\phi(a) h=P \tilde{\phi}(a) h$, where $P: \tilde{H} \rightarrow H$ is the orthogonal projection. It follows immediately that $\tilde{\phi}(a) h \in H$ for any $a \in \mathfrak{U}_{0}$ and $h \in H_{0}$. The same fact holds for any $a \in \mathfrak{U}$ and $h \in H$ due to the linearity and continuity. It means that $\tilde{\phi}$ is a trivial extension. Therefore $\phi$ admits only trivial extensions i.e. is nonextendible.

(b) $\Rightarrow$ (d). It follows immediately from (2.7) that any linear functional $f$ on $\mathfrak{A} \otimes H$ continuous with respect to $\|\cdot\|_{\phi}$ is of the form $f(\alpha)=f^{\prime}(\phi(\alpha))$, where $f^{\prime}$ is a continuous functional on $H$. Now, using the Riesz theorem (cf. [13] Ch. II, p. 50) one gets (d).

(d) $\Rightarrow$ (e). Assume that $\alpha \in \mathfrak{A} \otimes H$ and $\|\alpha\|_{\phi} \neq 0$. Then there exists a linear functional $f$, continuous with respect to $\|\cdot\|_{\phi}$, such that $f(\alpha) \neq 0$. According to (d): $f(\alpha)=(y \mid \phi(\alpha))$. Therefore $\phi(\alpha) \neq 0$.

(e) $\Rightarrow$ (b). Assume (e). Let $\alpha \in \mathfrak{A} \otimes H$. Then $\alpha-1 \otimes \phi(\alpha)$ belongs to the kernel of $(2.10)$ and therefore

$$
\|\alpha-1 \otimes \phi(\alpha)\|_{\phi}=0 \text {. }
$$

Now, using (2.5) we have

$$
\begin{aligned}
\|\alpha\|_{\phi} & =\|1 \otimes \phi(\alpha)+\alpha-1 \otimes \phi(\alpha)\|_{\phi} \\
& =\|1 \otimes \phi(\alpha)\|_{\phi}=\|\phi(\alpha)\| .
\end{aligned}
$$

The method of elementary extensions is not sufficient to construct nonextendible extensions of any positive map. Indeed this method (applied repeatedly) produces only finite extensions. Infinite extensions can be constructed by means of inductive limits.

Proposition 2.3. Let $\left(\mathfrak{U}, \mathfrak{U}_{+}, 1\right)$ be an ordered vector space with the unity and $\left\{\phi_{\omega}\right\}_{\omega \in \Omega}$ be a family of normalized positive maps defined on $\mathfrak{A}$. We assume that this family is directed i.e.: for any $\omega, \omega^{\prime} \in \Omega$ there exists $\omega^{\prime \prime} \in \Omega$ such that $\phi_{\omega} \subset \phi_{\omega^{\prime \prime}}$ and $\phi_{\omega^{\prime}} \subset \phi_{\omega^{\prime \prime}}$

Then there exists a normalized positive map $\phi_{\infty}$ such that

1. $\phi_{\omega} \subset \phi_{\infty}$ for any $\omega \in \Omega$.

2. $\phi_{\infty}$ is the smallest positive map satisfying the Condition 1. (i.e. any other is an extension of $\phi_{\infty}$ ). 
Proof. Let $H_{\omega}$ be the carrier Hilbert space of $\phi_{\omega}$. Clearly the family $\left\{H_{\omega}\right\}_{\omega \in \Omega}$ is directed by inclusion. Therefore $\bigcup_{\omega} H_{\omega}$ carries the natural pre-Hilbert space structure. Let $H_{\infty}=\lim$ ind $H_{\omega}$ denotes the completion of $\bigcup_{\omega} H_{\omega}$.

For any $a \in \mathfrak{A}$ and $h, k \in \bigcup_{\omega} H_{\omega}$ we put

$$
\left(k \mid \phi_{\infty}(a) h\right)=\left(k \mid \phi_{\omega}(a) h\right)_{H_{\omega}}
$$

where $\omega \in \Omega$ is chosen in such a way that $k, h \in H_{\omega}$. Evidently the RHS of (2.12) does not depend on $\omega$.

The Formula (2.12) defines $\phi_{\infty}(a)$ as a sesquilinear form on $\bigcup_{\omega} H_{\omega}$. If $a=a^{*} \in \mathfrak{A}$, then for sufficiently large positive $\lambda:-\lambda 1 \leqq a \leqq \lambda 1$ [cf. (1.4)]. Therefore $-\lambda I \leqq$ $\phi_{\omega}(a) \leqq \lambda I,\left\|\phi_{\omega}(a)\right\| \leqq \lambda$ and in virtue of (2.12) we have

$$
\left|\left(k \mid \phi_{\infty}(a) h\right)\right| \leqq \lambda\|k\|\|h\| .
$$

This inequality proves that $\phi_{\infty}(a)$ exists as an operator acting on $H_{\infty}$. The corresponding result for any $a \in \mathfrak{Q}$ follows from linearity.

Clearly

$$
\phi_{\infty}:\left(\mathfrak{l}, \mathfrak{U}_{+}, 1\right) \rightarrow\left(B\left(H_{\infty}\right) . B_{+}\left(H_{\infty}\right), I\right)
$$

and $\phi_{\infty} \supset \phi_{\omega}$ for any $\omega \in \Omega$. It is also evident that $\phi_{\infty}$ is the smallest extension of all $\phi_{\omega}$.

Q.E.D.

Remarks. 1. The normalized positive map satisfying the conditions 1 and 2 of Proposition 2.3 is called the inductive limit of the family $\left\{\phi_{\omega}\right\}_{\omega \in \Omega}$ and will be denoted by $\operatorname{limind}_{\omega \in \Omega} \phi_{\omega}$.

2. If a family $\left\{\phi_{\omega}\right\}_{\omega \in \Omega}$ contains the largest element $\phi_{\omega_{\max }}$, then

$$
\operatorname{limind}_{\omega \in \phi} \phi_{\omega}=\phi_{\omega_{\max }} \text {. }
$$

Now we are ready to prove our main theorem Proof of Theorem 1.7. Let

$$
\phi:\left(\mathfrak{U}, \mathfrak{U}_{+}, 1\right) \rightarrow\left(B(H), B_{+}(H), I\right) .
$$

We choose total subsets $\mathfrak{A}_{0} \subset \mathfrak{A}$ and $H_{0} \subset H$.

Let $\tilde{\Omega}$ be the set of all finite sequences of the form $\left(h, a_{1}, a_{2}, \ldots, a_{n}\right)$ where $h \in H_{0}, a_{i} \in \mathfrak{A}_{0}$ and $n \geqq 0$. The subset of $\tilde{\Omega}$ containing all sequences of the length $\geqq 2$ (i.e. $n \geqq 1$ ) will be denoted by $\Omega$.

If $\omega$ is an $n$-element sequence belonging to $\tilde{\Omega}$ and $a \in \mathfrak{A}_{0}$, then $\omega a$ denotes the $(n+1)$-element sequence obtained from $\omega$ by adding the element $a$ in the last place. Clearly $\omega a \in \Omega$.

Let us introduce in $\tilde{\Omega}$ an ordering relation $\leqq$ such that $(\tilde{\Omega}, \leqq)$ is a well ordered set and $\omega \leqq \omega a$ for any $\omega \in \tilde{\Omega}$ and $a \in \mathfrak{A}_{0}$. We refer to [7] for the theory of well ordered sets and the transfinite recursion. 
By using the transfinite recursion we shall introduce a family of normalized positive maps $\left\{\phi_{\omega}\right\}_{\omega \in \Omega}$ and a family of vectors $\left\{k_{\omega}\right\}_{\omega \in \tilde{\Omega}}$ such that:

1. $\phi \subset \phi_{\omega} \subset \phi_{\omega^{\prime}}$ for any $\omega, \omega^{\prime} \in \Omega$ such that $\omega \leqq \omega^{\prime}$.

2. $\left\{k_{\omega^{\prime}}: \omega^{\prime} \in \tilde{\Omega}\right.$ and $\left.\omega^{\prime} \leqq \omega\right\}$ is a total subset of the carrier Hilbert space $H_{\omega}$ of $\phi_{\omega}$.

3. $\left\|a \otimes k_{\omega^{\prime}}\right\|_{\phi_{\omega^{\prime} a}}=\left\|\phi_{\omega^{\prime} a}(a) k_{\omega^{\prime}}\right\|$

for any $a \in \mathfrak{U}_{0}$ and $\omega^{\prime} \in \tilde{\Omega}$.

At first we define $k_{\omega}$ for one-element sequences

$k_{(h)}=h$

for all $h \in H_{0}$.

Let $\omega_{1}$ be the first element of $\Omega$. Then $\omega_{1}$ is a two-element sequence $\omega_{1}=\left(h, a_{1}\right)$ where $h \in H_{0}$ and $a_{1} \in \mathfrak{U}_{0}$. According to (2.3) there exists an elementary extension $\phi_{\omega_{1}} \supset \phi$ such that

$$
\left\|a_{1} \otimes h\right\|_{\phi}=\left\|\phi_{\omega_{1}}\left(a_{1}\right) h\right\| .
$$

Moreover, since $\operatorname{dim} H_{\omega^{\prime}} / H \leqq 1$, one can choose a vector $k_{\omega_{1}} \in H_{\omega_{1}}$ such that $H_{\omega_{1}}$ is spanned by $H$ and $k_{\omega_{1}}$.

Now assume that $\phi_{\sigma}$ and $k_{\sigma}$ are already introduced for all $\sigma<\omega$, where $\omega$ is an element of $\Omega$. Let $\omega=\omega^{\prime} a$, where $\omega^{\prime} \in \tilde{\Omega}$ and $a \in \mathfrak{H}_{0}$. According to (2.3), there exists an elementary extension $\phi_{\omega} \supset \operatorname{limind}_{\sigma<\omega} \phi_{\sigma}$ such that

$$
\left\|a \otimes k_{\omega^{\prime}}\right\|_{\operatorname{limind}_{\sigma<\omega} \phi_{\sigma}}=\left\|\phi_{\omega}(a) k_{\omega^{\prime}}\right\| \text {. }
$$

Moreover, since $\operatorname{dim} H_{\omega} / \operatorname{limind}_{\sigma<\omega} H_{\sigma} \leqq 1$, one can choose a vector $k_{\omega}$ such that $H_{\omega}$ is spanned by $\lim _{\sigma<\omega} \operatorname{ind} H_{\sigma}$ and $k_{\omega}$.

According to the transfinite recursion principle, $k_{\sigma}$ and $\phi_{\omega}$ are introduced for all $\sigma \in \tilde{\Omega}$ and $\omega \in \Omega$. Properties 1. and 2. are obviously satisfied. The Equation (2.13) follows immediately from (2.14) and (2.6).

Now, we put

$$
\begin{aligned}
& \tilde{\phi}=\operatorname{limind}_{\omega \in \Omega} \phi_{\omega} \\
& \tilde{H}=\operatorname{limind}_{\omega \in \Omega} H_{\omega} .
\end{aligned}
$$

It is clear that $\left\{k_{\omega}: \omega \in \tilde{\Omega}\right\}$ is total in $\tilde{H}$. Moreover, using the second part of the Lemma 2.1 and (2.13) we get

$$
\left\|a \otimes k_{\omega}\right\|_{\tilde{\phi}}=\left\|\tilde{\phi}(a) k_{\omega}\right\|
$$

for all $a \in \mathfrak{U}_{0}$ and $\omega \in \tilde{\Omega}$. It means that $\tilde{\phi}$ satisfies the condition (C) of Proposition 2.2. Therefore $\tilde{\phi}$ is nonextendible extension of $\phi$.

If $\mathfrak{U}$ and $H$ are separable, then one may assume that $\mathfrak{U}_{0}$ and $H_{0}$ are denumerable. In this case $\Omega$ is denumerable and the space $H$ is separable.

This ends the proof of Theorem 1.7. 


\section{The Seminorm Associated with a Normalized Positive Map}

In the previous section we showed that the nonextendibility of a normalized positive map $\phi$ is equivalent to various other properties (cf. Prop. 2.2). Unfortunately these other properties are expressed in terms of the seminorm $\|\cdot\|_{\phi}$, which itself, according to the Definition (2.3) is closely related to the extension properties of $\phi$. It means that Proposition 2.2 is almost a tautology and no interesting applications can be expected.

In this section we give another definition of the seminorm associated with a normalized positive map. This definition seems to be more "explicite" and no extensions of $\phi$ are involved in it. We prove the equivalence of this new definition with (2.3).

As a result, Proposition 2.2 becomes meaningful. We derive a simple condition implying nonextendibility in the finite-dimensional case. Other useful applications are described in the following sections.

Let $\left(\mathfrak{U}, \mathfrak{A}_{+}, 1\right)$ be an ordered vector space with the unity, $H$ be a Hilbert space and

$$
\phi:\left(\mathfrak{U}, \mathfrak{A}_{+}, 1\right) \rightarrow\left(B(H), B_{+}(H), I\right) .
$$

Let $\alpha \in \mathfrak{A} \otimes H$. In this section

$$
\|\alpha\|_{\phi}=\left(\inf \sum_{i}\left(h_{i} \mid \phi\left(a_{i}\right) h_{i}\right)\right)^{1 / 2}
$$

where the infimum is taken over all finite subsets $\left\{\left(a_{i}, h_{i}\right)\right\}_{i=1,2, \ldots, n}$ of $\mathfrak{A} \times H$ such that $\alpha=\sum a_{i} \otimes h_{i}, a_{i} \in \mathfrak{U}_{+}$and $\sum a_{i} \leqq 1$.

At first we shall show that this formula defines a seminorm of $\mathfrak{A} \otimes H$.

Let $\alpha \in \mathfrak{A} \otimes H$ and $\lambda \in \mathbb{C}$. Assume that

$$
\begin{aligned}
& \alpha=\sum a_{i} \otimes h_{i} \\
& a_{i} \in \mathfrak{A}_{+}, \sum a_{i} \leqq 1 \\
& h_{i} \in H .
\end{aligned}
$$

Then $\lambda \alpha=\sum a_{i} \otimes \lambda h_{i}$ and according to (3.2)

$$
\begin{aligned}
\|\lambda \alpha\|_{\phi} & \leqq\left(\sum_{i}\left(\lambda h_{i} \mid \phi\left(a_{i}\right) \lambda h_{i}\right)\right)^{1 / 2} \\
& =|\lambda|\left(\sum\left(h_{i} \mid \phi\left(a_{i}\right) h_{i}\right)\right)^{1 / 2} .
\end{aligned}
$$

Taking the infimum of the RHS over all finite subsets $\left\{\left(a_{i}, h_{i}\right)\right\}_{i=1,2, \ldots, n} \subset \mathfrak{A} \times H$ satisfying (3.3) we get

$$
\|\lambda \alpha\|_{\phi} \leqq|\lambda|\|\alpha\|_{\phi} .
$$

Replacing in this inequality $\alpha$ and $\lambda$ by $\lambda \alpha$ and $\lambda^{-1}$ respectively and multiplying both sides by $|\lambda|$ we get the opposite inequality. Therefore

$$
\|\lambda \alpha\|_{\phi}=|\lambda|\|\alpha\|_{\phi} .
$$


To prove the triangle inequality we consider two elements $\alpha, \beta \in \mathfrak{U} \otimes H$. Assume that

$$
\begin{aligned}
& \alpha=\sum a_{i} \otimes h_{i} \\
& a_{i} \in \mathfrak{A}_{+}, \sum a_{i} \leqq 1 \\
& h_{i} \in H
\end{aligned}
$$

and

$$
\begin{aligned}
& \beta=\sum b_{j} \otimes g_{j} \\
& b_{j} \in \mathfrak{U}_{+}, \sum b_{j} \leqq 1 \\
& g_{j} \in H .
\end{aligned}
$$

Then for any $\lambda, \mu \in \mathbb{R}$ such that $\lambda, \mu>0, \lambda+\mu=1$ we have

$$
\begin{aligned}
& \alpha+\beta=\sum_{i} \lambda a_{i} \otimes \lambda^{-1} h_{i}+\sum_{j} \mu b_{j} \otimes \mu^{-1} g_{j} \\
& \lambda a_{i}, \mu b_{j} \in \mathfrak{U}_{+}, \sum_{i} \lambda a_{i}+\sum_{j} \mu b_{j} \leqq 1 \\
& \lambda^{-1} h_{i}, \mu^{-1} g_{j} \in H .
\end{aligned}
$$

Therefore according to (3.2)

$$
\begin{aligned}
\|\alpha+\beta\|_{\phi}^{2} & \leqq \sum_{i}\left(\lambda^{-1} h_{i} \mid \phi\left(\lambda a_{i}\right) \lambda^{-1} h_{i}\right)+\sum_{j}\left(\mu^{-1} g_{j} \mid \phi\left(\mu b_{j}\right) \mu^{-1} g_{h}\right) \\
& =\lambda^{-1} \sum_{i}\left(h_{i} \mid \phi\left(a_{i}\right) h_{i}\right)+\mu^{-1} \sum_{j}\left(g_{i} \mid \phi\left(b_{j}\right) g_{j}\right) .
\end{aligned}
$$

Taking the infimum of the RHS over all finite subsets $\left\{\left(a_{i}, h_{i}\right)\right\}_{i=1 \ldots n} \subset \mathfrak{A} \times H$ and $\left\{\left(b_{j}, g_{j}\right)\right\}_{j=1,2, \ldots, m} \subset \mathfrak{U} \times H$ satisfying (3.5) and (3.6) respectively we get

$$
\|\alpha+\beta\|_{\phi}^{2} \leqq \lambda^{-1}\|\alpha\|_{\phi}^{2}+\mu^{-1}\|\beta\|_{\phi}^{2} .
$$

Now, setting $\lambda=\|\alpha\|_{\phi} /\left(\|\alpha\|_{\phi}+\|\beta\|_{\phi}\right)$ and $\mu=\|\beta\|_{\phi} /\left(\|\alpha\|_{\phi}+\|\beta\|_{\phi}\right)$ we get immediately

$$
\|\alpha+\beta\|_{\phi} \leqq\|\alpha\|_{\phi}+\|\beta\|_{\phi} .
$$

This way we proved

Proposition 3.1. For any positive map (3.1), the function $\|\cdot\|_{\phi}$ introduced by (3.2) is a seminorm on $\mathfrak{U} \otimes H$.

Let us note that for any $a \in \mathfrak{A}_{+}$and $h \in H$ we have

$$
\|a \otimes h\|_{\phi}^{2} \leqq C(h \mid \phi(a) h)
$$

where $C=\inf \{\lambda \in \mathbb{R}: \lambda 1 \geqq a\}$. If $C=1$, then the formula follows directly from (3.2); if $C \neq 1$, one may apply (3.4).

The relation of the seminorm $\|\cdot\|_{\phi}$ to the extension problem is described in the following proposition. 
Proposition 3.2. Let

$$
f: \mathfrak{A} \otimes H \rightarrow \mathbb{C}
$$

be a linear functional. Then the following conditions are equivalent:

(a) $|f(\alpha)| \leqq\|\alpha\|_{\phi}$

for any $\alpha \in \mathfrak{A} \otimes H$.

(b) There exist an elementary extension $\phi_{1} \supset \phi$ and a normalized vector $y \in H_{1}\left(H_{1}\right.$ denotes the carrier Hilbert space of $\left.\phi_{1}\right)$ such that

$$
f(a \otimes h)=\left(y \mid \phi_{1}(a) h\right)
$$

for any $a \in \mathfrak{A}$ and $h \in H$.

Proof. (a) $\Rightarrow$ (b). Let

$$
\omega: \mathfrak{U} \rightarrow \mathbb{C}
$$

be a linear functional such that

$$
\omega(1)=1
$$

and

$$
|f(a \otimes h)|^{2} \leqq \omega(a)(h \mid \phi(a) h)
$$

for all $a \in \mathfrak{A}_{+}$and $h \in H$. We shall show later that the existence of such a functional is implied by (3.8).

As the carrier Hilbert space of the extended map $\phi_{1}$ we take the Hilbert space spanned by $H$ and a normalized vector $y$ such that

$$
(y \mid h)_{H_{1}}=f(1 \otimes h) \text {. }
$$

One can easily show that such a Hilbert space exists iff $|f(1 \otimes h)|^{2} \leqq(h \mid h)$. The last relation follows immediately from (3.12) (set $a=1$ ).

The extended map $\phi_{1}$ will be defined in terms of matrix elements

$$
\begin{aligned}
& \left(k \mid \phi_{1}(a) h\right)=(k \mid \phi(a) h), \\
& \left(y \mid \phi_{1}(a) h\right)=f(a \otimes h), \\
& \left(k \mid \phi_{1}(a) y\right)=\overline{f\left(a^{*} \otimes k\right),} \\
& \left(y \mid \phi_{1}(a) y\right)=\omega(a)
\end{aligned}
$$

for all $a \in \mathfrak{A}$ and $k, h \in H$. Comparing these expressions with (3.13) and (3.11) we get

$$
\phi_{1}(1)=I_{H_{1}} .
$$

Let $a \in \mathfrak{A}_{+}$. Then for any vector $h_{1}=h+\lambda y$ (where $h \in H, \lambda \in \mathbb{C}$ ) belonging to $H_{1}$ we have

$$
\left(h_{1} \mid \phi_{1}(a) h_{1}\right)=\left\{\begin{array}{l}
(h \mid \phi(a) h)+\bar{\lambda} f(a \otimes h) \\
+\lambda \overline{f(a \otimes h)}+\lambda \bar{\lambda} \omega(a) .
\end{array}\right.
$$


In virtue of (3.12), the RHS of the above equation in non-negative. This shows that

$$
\left(h_{1} \mid \phi_{1}(a) h_{1}\right) \geqq 0
$$

for all $a \in \mathfrak{U}_{+}$. Therefore the mapping

$$
\phi_{1}: \mathfrak{U} \rightarrow B\left(H_{1}\right)
$$

satisfies all requirements of the condition (b):

1. $\phi_{1}$ is a normalized positive map [cf. (3.18) and (3.19)].

2. $\phi_{1}$ is an extension of $\phi$ [cf. (3.14)].

3. This extension is elementary one: $\operatorname{dim} H_{1} / H \leqq 1$.

4. The formula (3.9) holds [cf. (3.15)].

Remark. Formulae (3.14)-(3.19) define $\phi_{1}(a)$ as a sesquilinear form on $H_{1}$. In order to show that $\phi_{1}(a)$ exists as an operator one has to prove some estimate. In our case this can be easily done:

If $a \in \mathfrak{U}_{+}$, then for sufficiently large positive $\lambda, \lambda 1-a \in \mathfrak{U}_{+}$[cf. (1.2)]. In virtue of (3.19) we have

$$
\left(h_{1} \mid \phi_{1}(1 \lambda-a) h_{1}\right) \geqq 0
$$

for any $h_{1} \in H_{1}$. Now, using (3.18) we get

$$
0 \leqq\left(h_{1} \mid \phi_{1}(a) h_{1}\right) \leqq \lambda\left(h_{1} \mid h_{1}\right) \text {. }
$$

This inequality proves that $\phi_{1}(a)$ exists as a bounded operator. The corresponding fact for any $a \in \mathfrak{U}$ follows now from linearity.

To end this part of the proof we have to show that one can find a linear functional (3.10) satisfying (3.11) and (3.12).

Assume (3.8). For any $b \in \mathfrak{U}_{+}$we put

$$
\Omega(b)=\sup \sum_{i}\left[\left|f\left(a_{i} \otimes h_{i}\right)\right|^{2} /\left(h_{i} \mid \phi\left(a_{i}\right) h_{i}\right)\right]
$$

where supremum is taken over all finite subsets $\left\{\left(a_{i}, h_{i}\right)\right\}_{i=1,2, \ldots, n} \subset \mathfrak{A} \times H$ such that

$$
\begin{aligned}
& a_{i} \in \mathfrak{U}_{+}, \sum a_{i} \leqq b \\
& \left(h_{i} \mid \phi\left(a_{i}\right) h_{i}\right) \neq 0 .
\end{aligned}
$$

If there is no finite subset of $\mathfrak{A} \times H$ satisfying (3.21), we put $\Omega(b)=0$.

It follows immediately from (3.20) that

$$
\begin{aligned}
& \Omega(b) \geqq 0 \\
& \Omega(\lambda b)=\lambda \Omega(b), \\
& \Omega(a+b) \geqq \Omega(a)+\Omega(b)
\end{aligned}
$$

for any $a, b \in \mathfrak{U}_{+}$and $\lambda \geqq 0$. Moreover

$$
|f(a \otimes h)|^{2} \leqq \Omega(a)(h \mid \phi(a) h)
$$

for any $a \in \mathfrak{A}_{+}$and $h \in H$. Indeed, if $(h \mid \phi(a) h) \neq 0$, then (3.24) is implied directly by (3.20). If $(h \mid \phi(a) h)=0$ then $\|a \otimes h\|_{\phi}=0$ [cf. (3.7)] and $f(a \otimes h)=0$ in virtue of (3.8). 
We shall show that

$$
\Omega(1) \leqq 1 \text {. }
$$

To this end take any finite subset $\left\{\left(a_{i}, h_{i}\right)\right\} \subset \mathfrak{U} \times H$ such that

$$
\begin{aligned}
& a_{i} \in \mathfrak{U}_{+}, \sum a_{i} \leqq 1 \\
& \left(h_{i} \mid \phi\left(a_{i}\right) h_{i}\right) \neq 0 .
\end{aligned}
$$

Let

$$
k_{i}=\left(\overline{f\left(a_{i} \otimes h_{i}\right)} /\left(h_{i} \mid \phi\left(a_{i}\right) h_{i}\right)\right) h_{i} .
$$

In virtue of (3.8) and (3.2) we have

$$
\begin{aligned}
& \left|\sum_{i} f\left(a_{i} \otimes k_{i}\right)\right|^{2}=\left|f\left(\sum a_{i} \otimes k_{i}\right)\right|^{2} \\
& \leqq\left\|\sum a_{i} \otimes k_{i}\right\|_{\phi}^{2} \leqq \sum\left(k_{i} \mid \phi\left(a_{i}\right) k_{i}\right) .
\end{aligned}
$$

Inserting here (3.27) we get

$$
\left|\sum_{i}\left[\left|f\left(a_{i} \otimes h_{i}\right)\right|^{2} /\left(h_{i} \mid \phi\left(a_{i}\right) h_{i}\right)\right]\right|^{2} \leqq \sum_{i}\left[\left|f\left(a_{i} \otimes h_{i}\right)\right|^{2} /\left(h_{i} \mid \phi\left(a_{i}\right) h_{i}\right)\right] .
$$

Therefore

$$
\sum_{i}\left[\left|f\left(a_{i} \otimes h_{i}\right)\right|^{2} /\left(h_{i} \mid \phi\left(a_{i}\right) h_{i}\right)\right] \leqq 1 .
$$

Taking the supremum of the LHS over all finite subsets $\left\{\left(a_{i}, h_{i}\right)\right\}_{i=1 \ldots n} \subset \mathfrak{U} \times H$ satisfying (3.26) we get (3.25).

Let us consider the set

$$
Z_{\Omega}=\left\{a \in \mathfrak{U}_{+}: \Omega(a) \geqq 1\right\} .
$$

In virtue of (3.22) and (3.23), $Z_{\Omega}$ is convex. Moreover according to (3.25), 1 is not an interior point of $Z_{\Omega}$ (here we consider $Z_{\Omega}$ as a subset of $\mathfrak{A}_{r}$ ).

Assume for the moment that $Z_{\Omega}$ is not empty: $b \in Z_{\Omega}$. Then for any $a \in \mathfrak{A}_{+}$ we have $\Omega(a+b) \geqq \Omega(a)+\Omega(b) \geqq \Omega(b) \geqq 1$. It means that

$$
b+\mathfrak{U}_{+} \subset Z_{\Omega} .
$$

Therefore $Z_{\Omega}$ contains interior points and we may apply the well known separation theorem (see e.g. [5], Ch. 1, p. 24, Th. 3). It says that there exists a (closed) hyperplane $\mathfrak{A}_{0} \subset \mathfrak{U}_{r}$ passing through 1 such that $Z_{\Omega}$ lies on one side of $\mathfrak{A}_{0}$.

Let $\omega: \mathfrak{Q}_{r} \rightarrow \mathbb{R}$ be the linear functional defining $\mathfrak{U}_{0}$ :

$$
\mathfrak{U}_{0}=\left\{a \in \mathfrak{U}_{r}: \omega(a)=1\right\} \text {. }
$$

Then

$$
\omega(1)=1 \text {. }
$$

In virtue of (1.2) and (3.29), $\lambda 1 \in Z_{\Omega}$ for sufficiently large positive $\lambda$. Therefore, at least for some points $a \in Z_{\Omega}$ we have $\omega(a)>1$. Since $Z_{\Omega}$ lies on one side of $\mathfrak{U}_{0}$, we have $\omega(a) \geqq 1$ for all $a \in Z_{\Omega}$. Now, taking into account (3.28) and (3.22) we get

$$
\omega(a) \geqq \Omega(a)
$$

for any $a \in \mathfrak{U}_{+}$. Combining this inequality with (3.24) we finally obtain (3.12). 
If $Z_{\Omega}$ is empty, then $\Omega(\cdot)$ and $f(\cdot)$ vanish identically and relations (3.11) and (3.12) are satisfied by any normalized positive functional $\omega$.

(b) $\Rightarrow$ (a). Assume (3.9). Let $\alpha \in \mathfrak{U} \otimes H$ and

$$
\alpha=\sum_{i=1}^{n} a_{i} \otimes h_{i}
$$

where

$$
\begin{aligned}
& a_{i} \in \mathfrak{U}_{+}, \sum a_{i} \leqq 1 \\
& h_{i} \in H .
\end{aligned}
$$

Using the Cauchy-Schwarz inequality in the two different forms (first for positive sesquilinear forms on $H_{1}$, second for scalar product in $\mathbb{C}^{n}$ ) we have

$$
\begin{aligned}
& |f(\alpha)|^{2} \leqq\left(\sum\left|f\left(a_{i} \otimes h_{i}\right)\right|\right)^{2}=\left(\sum \mid\left(y\left|\phi_{1}\left(a_{i}\right) h_{i}\right|\right)^{2}\right. \\
& \leqq\left(\sum\left(y \mid \phi_{1}\left(a_{i}\right) y\right)^{1 / 2}\left(h_{i} \mid \phi_{1}\left(a_{i}\right) h_{i}\right)^{1 / 2}\right)^{2} \leqq\left(\sum\left(y \mid \phi_{1}\left(a_{i}\right) y\right)\right)\left(\sum\left(h_{i} \mid \phi_{1}\left(a_{i}\right) h_{i}\right)\right) \\
& \leqq(y \mid y) \sum\left(h_{i} \mid \phi_{1}\left(a_{i}\right) h_{i}\right) .
\end{aligned}
$$

Now we may replace $(y \mid y)$ by 1 ( $y$ is a normalized vector) and $\left(h_{i} \mid \phi_{1}\left(a_{i}\right) h_{i}\right)$ by $\left(h_{i} \mid \phi\left(a_{i}\right) h_{i}\right)\left(\phi_{1} \supset \phi\right.$ and $\left.h_{i} \in H\right)$. Taking the infimum of the RHS over all finite subsets $\left\{\left(a_{i}, h_{i}\right)\right\} \subset \mathfrak{A} \times H$ satisfying (3.30) and (3.31) we get (3.8).

Q.E.D.

Now we are able to show that our Definition (3.2) is equivalent to the Definition (2.3) used in the previous section:

$$
\|\alpha\|_{\phi}=\max _{\phi_{1} \in \mathrm{el}(\phi)}\left\|\phi_{1}(\alpha)\right\| \text {. }
$$

To this end we note that

$$
\|\alpha\|_{\phi}=\max |f(\alpha)|
$$

where $f$ runs over the set of all functionals satisfying the condition (3.8) (HahnBanach theorem) and

$$
\left\|\phi_{1}(\alpha)\right\|=\max \left|\left(y \mid \phi_{1}(\alpha)\right)\right|
$$

where $y$ runs over the set of all normalized vectors in $H_{1}$. Now (3.32) follows directly from Proposition 3.2.

Combining Proposition 2.2 and (3.2) we get the following sufficient condition for nonextendibility in the finite dimensional case.

Theorem 3.3. Let $\left(\mathfrak{H}, \mathfrak{U}_{+}, 1\right)$ be a finite-dimensional ordered vector space with the unity, $H$ be a finite-dimensional Hilbert space and

$$
\phi:\left(\mathfrak{U}, \mathfrak{U}_{+}, 1\right) \rightarrow\left(B(H), B_{+}(H), I\right) .
$$

Assume that the subspace $N \subset \mathfrak{U} \otimes H$ spanned by all $a \otimes h$ such that $a \in \mathfrak{U}_{+}$and $\phi(a) h=0$ is $\operatorname{dim} H(\operatorname{dim} \mathfrak{U}-1)$ dimensional:

$$
\operatorname{dim} N=\operatorname{dim} H(\operatorname{dim} \mathfrak{A}-1) .
$$

Then $\phi$ is nonextendible. 
Proof. Clearly $N$ is contained in the kernel of the mapping $\phi: \mathfrak{A} \otimes H \rightarrow H$. The dimension of this kernel can be easily calculated and equals $\operatorname{dim} \mathfrak{A} \operatorname{dim} H-$ $\operatorname{dim} H=\operatorname{dim} N$. Therefore $N$ coincides with this kernel.

On the other hand, in virtue of (3.7) $\|\alpha\|_{\phi}=0$ for all $\alpha \in N$. It means that $N \subset N_{\phi}$ (for the definition of $N_{\phi}$ see Proposition 2.2 condition (e)).

Now, the nonextendibility of $\phi$ follows directly from Proposition 2.2. Q.E.D.

Let $H^{\prime}$ be the subspace generated by all $h \in H$ such that we have $\phi(a) h=0$ for some $a \in \mathfrak{A}_{+}, a \neq 0$. Let us note that $N$ is contained in the kernel of the mapping $\phi: \mathfrak{U} \otimes H^{\prime} \rightarrow H$.

Since $\phi(1)=I$, the image of this mapping contains $H^{\prime}$. Therefore $\operatorname{dim} N \leqq \operatorname{dim} H^{\prime}$ $(\operatorname{dim} \mathfrak{U}-1)$. It shows that (3.33) can be satisfied only if $H^{\prime}=H$.

\section{Nonextendible Maps of $C^{*}$-Algebras}

In this section $\mathfrak{A}$ is a $C^{*}$-algebra, $\mathfrak{A}_{+}$denotes the cone of positive elements of $\mathfrak{A}$ and 1 denotes the unity of $\mathfrak{A}$. It is well known that $\left(\mathfrak{U}, \mathfrak{U}_{+}, 1\right)$ is an ordered vector space with the unity.

Let

$$
\phi:\left(\mathfrak{H}, \mathfrak{U}_{+}, 1\right) \rightarrow\left(B(H), B_{+}(H), I\right) .
$$

Then we have (cf. [18])

$$
\phi\left(a^{2}\right)-\phi(a)^{2} \geqq 0
$$

for any selfadjoint element $a \in \mathfrak{U}$. This important property of positive maps of $C^{*}$-algebras is known as the Kadison inequality.

Theorem 4.1. Assume that (4.1) is a Jordan representation, i.e. $\phi\left(a^{2}\right)=\phi(a)^{2}$ for any $a \in \mathfrak{U}$. Then $\phi$ is nonextendible.

Proof. Let $\tilde{\phi} \supset \phi$. Then

$$
\phi(a)=P \tilde{\phi}(a) P
$$

for any $a \in \mathfrak{U}$. Here $P$ denotes the orthogonal projection of $\tilde{H}$ onto $H ; \tilde{H}$ is the carrier Hilbert space of $\tilde{\phi}$. Now, for any selfadjoint element $a \in \mathfrak{U}$ we have

$$
\begin{aligned}
0 & \leqq P \tilde{\phi}(a)\left(I_{\tilde{H}}-P\right) \tilde{\phi}(a) P=P \tilde{\phi}(a)^{2} P-\phi(a)^{2} \\
& =P \tilde{\phi}(a)^{2} P-\phi\left(a^{2}\right)=P \tilde{\phi}(a)^{2} P-P \tilde{\phi}\left(a^{2}\right) P \\
& =P\left(\tilde{\phi}(a)^{2}-\tilde{\phi}\left(a^{2}\right)\right) P .
\end{aligned}
$$

The last expression is non-positive in virtue of the Kadison inequality for $\tilde{\phi}$. Therefore $P \tilde{\phi}(a)\left(I_{\tilde{H}}-P\right) \tilde{\phi}(a) P=0$ and $\left(I_{\tilde{H}}-P\right) \tilde{\phi}(a) P=0$. It shows that $\tilde{\phi}(a) h \in H$ for any $h \in H$. This means that $\tilde{\phi}$ is a trivial extension of $\phi$.

Q.E.D.

Now, let us consider linear functionals

$$
f: \mathfrak{U} \otimes H \rightarrow \mathbb{C}
$$


such that

$$
|f(a \otimes h)|^{2} \leqq\left(h\left|\phi\left(a^{2}\right)-\phi(a)^{2}\right| h\right)
$$

for all selfadjoint $a \in \mathfrak{A}$ and all $h \in H$.

Refining the previous argument we get the following improved version of Theorem 4.1.

Theorem 4.2. Let (4.1) be a normalized positive map. Assume that $f=0$ is the only functional satisfying the estimate (4.4). Then (4.1) is nonextendible.

Proof. Let $\tilde{\phi} \supset \phi$. We use the notation introduced in the previous proof.

Let us fix a normalized vector $y \in \tilde{H} \ominus H$. For any $a \in \mathfrak{A}$ and $h \in H$ we put

$f(a \otimes h)=(y \mid \tilde{\phi}(a) h)$.

Then for selfadjoint $a$ we have

$$
\begin{aligned}
|f(a \otimes h)|^{2} & =(h \mid \tilde{\phi}(a) y)(y \mid \tilde{\phi}(a) h) \\
& \leqq\left(h \mid \tilde{\phi}(a)\left(I_{\tilde{H}}-P\right) \tilde{\phi}(a) h\right) \\
& =\left(h \mid \tilde{\phi}(a)^{2} h\right)-\left(h \mid \phi(a)^{2} h\right) .
\end{aligned}
$$

Now, using the Kadison inequality for $\tilde{\phi}$ we get

$$
\begin{aligned}
|f(a \otimes h)|^{2} & \leqq\left(h \mid \tilde{\phi}\left(a^{2}\right) h\right)-\left(h \mid \phi(a)^{2} h\right) \\
& =\left(h\left|\phi\left(a^{2}\right)-\phi(a)^{2}\right| h\right) .
\end{aligned}
$$

This shows that the linear functional $f$ introduced by (4.5) satisfies the estimate (4.4). We assumed that any such a functional vanishes identically. Therefore

$(y \mid \tilde{\phi}(a) h)=0$.

Since this result holds for every $y \in \tilde{H} \ominus H$, we have $\tilde{\phi}(a) h \in H$ and the extension $\tilde{\phi} \supset \phi$ must be trivial.

Q.E.D.

We do not know, whether the statement converse to Theorem 4.2 holds in general. We have however

Theorem 4.3. Assume that $\operatorname{dim} \mathfrak{U}<\infty$ and that (4.1) is nonextendible. Then $f=0$ is the only functional on $\mathfrak{A} \otimes H$ satisfying (4.4).

Proof. At first we note that $\mathfrak{A}$ is a finite direct sum of matrix algebras. Therefore there exists a linear functional

$$
\operatorname{Tr}: \mathfrak{U} \rightarrow \mathbb{C}
$$

such that $\operatorname{Tr} e=1$ for every minimal non-zero projection $e$. Since any positive element of $\mathfrak{A}$ can be written as a linear combination of mutually orthogonal minimal projections with positive coefficients, we get

$$
a^{2} \leqq a \operatorname{Tr} a
$$

for all $a \in \mathfrak{U}_{+}$.

Assume now that (4.3) satisfies (4.4). Then

$$
|f(a \otimes h)|^{2} \leqq\left(h \mid \phi\left(a^{2}\right) h\right)
$$

for all $a \in \mathfrak{U}_{+}$and $h \in H$. 
Let $\alpha \in \mathfrak{U} \otimes H$ and

$$
\alpha=\sum a_{i} \otimes h_{i}
$$

where

$$
\begin{aligned}
& a_{i} \in \mathfrak{U}_{+}, \sum a_{i} \leqq 1 \\
& h_{i} \in H .
\end{aligned}
$$

Using the Cauchy-Schwarz inequality, (4.7) and (4.6) we have

$$
\begin{aligned}
|f(\alpha)|^{2} & =\left|\sum f\left(a_{i} \otimes h_{i}\right)\right|^{2} \leqq\left(\sum \operatorname{Tr} a_{i}\right)\left(\sum\left|f\left(a_{i} \otimes h_{i}\right)\right|^{2} / \operatorname{Tr} a_{i}\right) \\
& \leqq(\operatorname{Tr} 1) \sum\left(\left(h_{i} \mid \phi\left(a_{i}^{2}\right) h_{i}\right) / \operatorname{Tr} a_{i}\right) \leqq(\operatorname{Tr} 1) \sum\left(h_{i} \mid \phi\left(a_{i}\right) h_{i}\right) .
\end{aligned}
$$

Now, taking the infimum of the last expression over all finite subsets $\left\{\left(a_{i}, h_{i}\right)\right\} \subset$ $\mathfrak{U} \times H$ satisfying (4.8) and (4.9) we get [cf. (3.2)]

$$
|f(\alpha)| \leqq(\operatorname{Tr} 1)^{1 / 2}\|\alpha\|_{\phi} .
$$

According to Proposition 2.2 (d) there exists a vector $y \in H$ such that

$$
f(a \otimes h)=(y \mid \phi(a) h)
$$

for all $a \in \mathfrak{A}$ and $h \in H$.

Setting $a=1$ in (4.4) we get $f(1 \otimes h)=0$. Formula (4.10) shows now that $y=0$ and $f=0$.

The Stinespring theorem says that any completely positive normalized map of a $C^{*}$-algebra can be extended to a representation. It follows immediately that any nonextendible, completely positive map is a representation. It is interesting that this result can be considerably improved.

Theorem 4.4. Assume that (4.1) is nonextendible and 2-positive. Then (4.1) is a representation, i.e. $\phi(a b)=\phi(a) \phi(b)$ for any $a, b \in \mathfrak{A}$.

Proof. We recall that $\phi$ is 2-positive if

$$
\left(\begin{array}{ll}
a, & b \\
c, & d
\end{array}\right) \geqq 0 \Rightarrow\left(\begin{array}{ll}
\phi(a), & \phi(b) \\
\phi(c), & \phi(d)
\end{array}\right) \geqq 0
$$

for any $a, b, c, d \in \mathfrak{U}$.

Let $a \in \mathfrak{U}_{+}$and $b \in \mathfrak{A}$. Then

$$
\left(\begin{array}{ll}
a, & a b^{*} \\
b a, & b a b^{*}
\end{array}\right) \geqq 0 .
$$

Therefore

$$
\left(\begin{array}{ll}
\phi(a), & \phi\left(a b^{*}\right) \\
\phi(b a), & \phi\left(b a b^{*}\right)
\end{array}\right) \geqq 0 .
$$

It means that for fixed $b$

$$
\mathfrak{U} \ni a \rightarrow\left(\begin{array}{ll}
\phi(a), & \phi\left(a b^{*}\right) \\
\phi(b a), & \phi\left(b a b^{*}\right)
\end{array}\right) \in B(H \oplus H)
$$


is a (non-normalized) positive map. We apply the normalization procedure. Let $Q$ denote the value of (4.11) for $a=1$ :

$$
Q=\left(\begin{array}{ll}
I, & \phi\left(b^{*}\right) \\
\phi(b), & \phi\left(b b^{*}\right)
\end{array}\right) .
$$

Let $\tilde{H}$ be the closure of $Q(H \oplus H)$. Then $Q$ in invertible on $\tilde{H}$ and we put

$$
\tilde{\phi}(a)=Q^{-1 / 2}\left(\begin{array}{ll}
\phi(a), & \phi\left(a b^{*}\right) \\
\phi(b a), & \phi\left(b a b^{*}\right)
\end{array}\right) Q^{-1 / 2} .
$$

One can easily show that (4.13) defines a bounded operator acting on $\tilde{H}$. Moreover

$$
\tilde{\phi}:\left(\mathfrak{A}, \mathfrak{A}_{+}, 1\right) \rightarrow\left(B(\tilde{H}), B_{+}(\tilde{H}), I\right) .
$$

Let

$$
i: H \rightarrow \tilde{H}
$$

be a linear map introduced by

$$
i(h)=Q^{1 / 2}\left(\begin{array}{l}
h \\
0
\end{array}\right) \text {. }
$$

Using (4.13) and (4.15) we get immediately

$$
(k \mid \phi(a) h)=(i(k) \mid \tilde{\phi}(a) i(h))
$$

for any $k, h \in H$ and $a \in \mathfrak{A}$. Setting here $a=1$ we prove that (4.14) is an isometric embeding. Now, the above formula means that $\tilde{\phi}$ is an extension of $\phi$ [cf. (1.7)]. We assumed that $\phi$ is nonextendible. Thus $\tilde{\phi} \supset \phi$ is a trivial extension. Therefore [cf. (1.8)] for any $h \in H$ we have:

$$
\tilde{\phi}(a) i(h)=i(\phi(a) h) \text {. }
$$

Using (4.13) and (4.15) one may rewrite this formula in the more explicite way:

$$
Q^{-1 / 2}\left(\begin{array}{ll}
\phi(a), & \phi\left(a b^{*}\right) \\
\phi(b a), & \phi\left(b a b^{*}\right)
\end{array}\right)\left(\begin{array}{l}
h \\
0
\end{array}\right)=Q^{1 / 2}\left(\begin{array}{c}
\phi(a) h \\
0
\end{array}\right)
$$

and.

$$
\left(\begin{array}{ll}
\phi(a), & \phi\left(a b^{*}\right) \\
\phi(b a), & \phi\left(b a b^{*}\right)
\end{array}\right)\left(\begin{array}{l}
h \\
0
\end{array}\right)=\left(\begin{array}{ll}
I, & \phi\left(b^{*}\right) \\
\phi(b), & \phi\left(b b^{*}\right)
\end{array}\right)\left(\begin{array}{c}
\phi(a) h \\
0
\end{array}\right) .
$$

In particular it means that

$$
\phi(b a) h=\phi(b) \phi(a) h .
$$

We know (cf. [18]) that any positive map of an abelian $C^{*}$-algebra is completely positive. Therefore we have

Theorem 4.5. Any normalized nonextendible positive map (4.1) of an abelian $C^{*}$ algebra is a representation.

The theorem corresponding to Theorem 4.4 holds also for copositive maps. We state it here without the proof. 
Theorem 4.6. Assume that (4.1) is nonextendible and 2-copositive. Then (4.1) is a corepresentation i.e. $\phi(a b)=\phi(b) \phi(a)$ for any $a, b \in \mathfrak{U}$.

Theorem 4.5 can be slightly generalized.

Theorem 4.7. Assume that (4.1) is nonextendible and that $b$ belongs to the centrum of $\mathfrak{U}$ (i.e. $b a=a b$ for all $a \in \mathfrak{U})$. Then

$$
\phi(b a)=\phi(b) \phi(a)
$$

and

$$
\phi(a) \phi(b)=\phi(b) \phi(a)
$$

for all $a \in \mathfrak{U}$.

Proof. Let $a \in \mathfrak{U}_{+}$. According to our assumption, the entries of the following matrix

$$
\left(\begin{array}{ll}
a, & a b^{*} \\
b a, & b a b^{*}
\end{array}\right) \geqq 0
$$

belong to an abelian $C^{*}$-subalgebra of $\mathfrak{A}$ (which is generated by these entries). Restricting for the moment $\phi$ to this subalgebra and remembering that positive maps of abelian algebras are 2-positive we get

$$
\left(\begin{array}{ll}
\phi(a), & \phi\left(a b^{*}\right) \\
\phi(b a), & \phi\left(b a b^{*}\right)
\end{array}\right) \geqq 0 .
$$

It means that also in this case the map (4.11) is positive. Repeating the reasoning following (4.11) we get (4.16). Now, (4.17) follows easily:

$$
\begin{aligned}
\phi(a) \phi(b) & =\left(\phi\left(b^{*}\right) \phi\left(a^{*}\right)\right)^{*}=\phi\left(b^{*} a^{*}\right)^{*} \\
& =\phi(a b)=\phi(b a)=\phi(b) \phi(a) .
\end{aligned}
$$

We would like to end this section with the following remark.

Let $\mathfrak{U}$ be a non-abelian $C^{*}$-algebra. It is known that the triple $\left(\mathfrak{U}, \mathfrak{U}_{+}, 1\right)$ does not determine the multiplication rule of $\mathfrak{A}$. More precisely one may introduce in the vector space $\mathfrak{A}$ at least two different multiplication rules (i.e. different $C^{*}$-algebra structure) which leads to the same cone of positive elements $\mathfrak{U}_{+}$and to the same unity 1 . The multiplication rule, which differs from the usual one by the order of factors is the best known example proving this statement.

We stress this property saying that $\left(\mathfrak{A}, \mathfrak{A}_{+}, 1\right)$ is not susceptible to the order of multiplication in $\mathfrak{A}$. The same can be said about the notion of positive map.

On the contrary, the notion of completely positive map and of $n$-positive map $(n>1)$ are susceptible to the order of multiplication.

Theorem 4.4 shows us that if a nonextendible normalized positive map satisfies some weak assumption susceptible to the order of multiplication, then it must be multiplicative. In the abelian case, where no question about the order of multiplication arises, any nonextendible normalized positive map is multiplicative. 


\section{Positive Maps of the Jordan Algebra $M_{2 s}$}

In this section we show that the general theory given in the Section 2 provides us with the complete description of the set of all positive maps of the simplest nonabelian Jordan algebra. It turns out that any nonextendible normalized positive map of this algebra is a Jordan representation.

Let $M_{2 s}$ be the set of all symmetric $2 \times 2$-matrices with complex entries.

$$
M_{2 s}=\left\{\left(\begin{array}{ll}
r, s \\
s, t
\end{array}\right): r, s, t \in \mathbb{C}\right\} \text {. }
$$

One can easily check that $M_{2 s}$ is a Jordan subalgebra of the matrix algebra $M_{2}$. The cone of symmetric positive matrices will be denoted by $M_{2 s+}$ and the unity matrix $\left(\begin{array}{ll}1 & 0 \\ 0 & 1\end{array}\right)$ by 1 . Clearly $\left(M_{2 s}, M_{2 s+}, 1\right)$ is an ordered vector space with the unity.

Let us note that extreme rays of $M_{2 s+}$ are generated by one-dimensional projections.

Let

$$
\phi:\left(M_{2 s}, M_{2 s+}, 1\right) \rightarrow\left(B(H), B_{+}(H), I\right) .
$$

Proposition 5.1. Let e and $e^{\prime}$ be two orthogonal one-dimensional projections belonging to $M_{2 s}$ and $k, k^{\prime} \in H$. Then

$$
(k \mid \phi(e) k) \leqq\left\|e \otimes k+e^{\prime} \oplus k^{\prime}\right\|_{\phi}^{2} .
$$

To prove this statement we need the following estimation

\section{Lemma 5.2. Let}

$$
\begin{aligned}
& t_{1}, t_{2}, \ldots, t_{n} \in \mathbb{R}, \\
& \alpha_{1}, \alpha_{2}, \ldots, \alpha_{n} \in \mathbb{R}, \alpha_{i} \geqq 0, \\
& h_{1}, h_{2}, \ldots, h_{n} \in H, \sum \alpha_{i} t_{i} h_{i}=0 .
\end{aligned}
$$

Then

$$
\left(\sum_{i} \alpha_{i}\right)^{-1}\left(h \mid \phi\left(\begin{array}{ll}
1 & 0 \\
0 & 0
\end{array}\right) h\right) \leqq \sum_{i} \alpha_{i}\left(h_{i} \mid \phi\left(\begin{array}{cc}
1, & t_{i} \\
t_{i}, & t_{i}^{2}
\end{array}\right) h_{i}\right)
$$

where $h=\sum_{i} \alpha_{i} h_{i}$.

Proof. Assume at first that $t_{i} \neq 0$ for all $i$ and $t_{i} \neq t_{j}$ for $i \neq j$. We put

$$
\begin{aligned}
& h(t)=\sum_{i}\left(\alpha_{i} h_{i} \prod_{j \neq i}\left(1-t / t_{j}\right)\right), \\
& u(t)=\left(h(t) \mid \phi\left(\begin{array}{ll}
1, & t \\
t, & t^{2}
\end{array}\right) h(t)\right),
\end{aligned}
$$

where $t$ is a real variable.

$h(t)$ is a vector valued polynomial with respect to the variable $t$. We have $h(t)=-\left(\sum_{i} \alpha_{i} t_{i} h_{i} / \prod_{j}\left(-t_{j}\right)\right) t^{n-1}+$ terms of smaller order. 
Now, using one of our assumptions one sees that $h(t)$ is a polynomial of the order $\leqq(n-2)$. Therefore $u(t)$ is a polynomial of the order $\leqq(2 n-2)$.

Let us notice that $\left(\begin{array}{ll}1, & t \\ t, & t^{2}\end{array}\right) \in M_{2 s+}$. It shows that $u(t) \geqq 0$ for all $t \in \mathbb{R}$. Therefore

$$
u(t)=\overline{v(t)} v(t)
$$

where $v(t)$ is a complex valued polynomial of the order $\leqq(n-1)$ [to obtain this factorization, one writes $u(t)$ as a product of order 1 polynomials and clusters the factors in a suitable way].

We shall use the Lagrange formula for the polynomial $v(t)$ expressing $v(t)$ for any $t$ in terms of the values in $n$ given points

$$
v(t)=\sum_{i}\left(v\left(t_{i}\right) \prod_{j \neq i}\left(1-t / t_{j}\right) /\left(1-t_{i} / t_{j}\right)\right) .
$$

In particular

$$
v(0)=\sum_{i}\left(v\left(t_{i}\right) / \prod_{j \neq i}\left(1-t_{i} / t_{j}\right)\right) .
$$

Setting $t=t_{i}$ in (5.4) we get

and

$$
h\left(t_{i}\right)=\alpha_{i} h_{i} \prod_{j \neq i}\left(1-t_{i} / t_{j}\right)
$$

$$
h_{i}=\left(h\left(t_{i}\right) / \alpha_{i}\right) \prod_{j \neq i}\left(1-t_{i} / t_{j}\right)^{-1} .
$$

Therefore, denoting by $R$ the $R H S$ of (5.3) we have

$$
R=\sum_{i}\left[\left(u\left(t_{i}\right) / \alpha_{i}\right) \prod_{j \neq i}\left(1-t_{i} / t_{j}\right)^{-2}\right]=\sum_{i} \bar{C}_{i} C_{i} / \alpha_{i}
$$

where

$$
C_{i}=v\left(t_{i}\right) \prod_{j \neq i}\left(1-t_{i} / t_{j}\right)^{-1} .
$$

Now using the relation

$$
\sum_{i}\left(\bar{C}_{i} C_{i} / \alpha_{i}\right) \geqq\left|\sum C_{i}\right|^{2} / \sum \alpha_{i}
$$

which can be easily derived from the Cauchy-Schwarz inequality and (5.6) we get

$$
R \geqq|v(0)|^{2} / \sum \alpha_{i}=u(0) / \sum \alpha_{i} .
$$

To end the proof we note that $u(0)$ coincides with $\left(h \mid \phi\left(\begin{array}{ll}1 & 0 \\ 0 & 0\end{array}\right) h\right)$.

If two $t_{i}$ coincide (say $t_{1}=t_{2}$ ) then (5.3) can be reduced to the case already considered:

$$
\begin{aligned}
& \alpha_{1}\left(h_{1} \mid \phi\left(\begin{array}{ll}
1, & t_{1} \\
t_{1}, & t_{1}^{2}
\end{array}\right) h_{1}\right)+\alpha_{2}\left(h_{2} \mid \phi\left(\begin{array}{ll}
1, & t_{1} \\
t_{1}, & t_{1}^{2}
\end{array}\right) h_{2}\right) \\
& \geqq\left(\alpha_{1}+\alpha_{2}\right)\left(h \mid \phi\left(\begin{array}{ll}
1, & t_{1} \\
t_{1}, & t_{1}^{2}
\end{array}\right) h\right)
\end{aligned}
$$


where $h=\left(\alpha_{1} /\left(\alpha_{1}+\alpha_{2}\right)\right) h_{1}+\left(\alpha_{2} /\left(\alpha_{1}+\alpha_{2}\right)\right) h_{2}$. This inequality follows immediately from the well known convexity of positive quadratic functionals.

If some $t_{i}$ vanishes (say $t_{1}=0$ ) then we separate the corresponding term in the $\operatorname{sum}(5.3)$ :

$$
R=\alpha_{1}\left(h_{1} \mid \phi\left(\begin{array}{ll}
1 & 0 \\
0 & 0
\end{array}\right) h_{1}\right)+\sum_{i \neq 1} \alpha_{i}\left(h_{i} \mid \phi\left(\begin{array}{ll}
t, & t_{i} \\
t_{i}, & t_{i}^{2}
\end{array}\right) h_{i}\right) .
$$

Now we may use (5.3) to estimate the second term (all $t_{i} \neq 0$ for $i>1$ ). We get

$$
R \geqq \alpha_{1}\left(h_{1} \mid \phi\left(\begin{array}{ll}
1 & 0 \\
0 & 0
\end{array}\right) h_{1}\right)+\left(\sum_{i \neq 1} \alpha_{1}\right)\left(h^{\prime} \mid \phi\left(\begin{array}{ll}
1 & 0 \\
0 & 0
\end{array}\right) h^{\prime}\right)
$$

where $h^{\prime}=\sum_{i \neq 1} \alpha_{i} h_{i} / \sum_{i \neq 1} \alpha_{i}$. Now, using once more the convexity of positive quadratic forms we get

$$
R \geqq\left(\sum_{i} \alpha_{i}\right)\left(h^{\prime \prime} \mid \phi\left(\begin{array}{ll}
1 & 0 \\
0 & 0
\end{array}\right) h^{\prime \prime}\right)
$$

where $h^{\prime \prime}=\sum_{i} \alpha_{i} h_{i} / \sum_{i} \alpha_{i}$. Clearly the RHS of the above inequality coincides with the LHS of (5.3). Therefore (5.3) is proved in full generality.

Q.E.D. Proof of Proposition 5.1. We assume that $e=\left(\begin{array}{ll}1, & 0 \\ 0, & 0\end{array}\right)$ and $e^{\prime}=\left(\begin{array}{ll}0, & 0 \\ 0, & 1\end{array}\right)$. The general case can be reduced to this particular one: the group of automorphisms of $M_{2 s}$ acts transitively in the set of all pairs of orthogonal one-dimensional projections.

Let

$$
\left\{\left(a_{i}, h_{i}\right)\right\}_{i=1,2, \ldots, n}
$$

be a finite subset of $M_{2 s} \times H$ such that

$$
\begin{aligned}
& a_{i} \in M_{2 s^{+}}, \sum a_{i} \leqq 1, \\
& \sum a_{i} \otimes h_{i}=e \otimes k+e^{\prime} \otimes k^{\prime} .
\end{aligned}
$$

We have to prove that

$$
(k \mid \phi(e) k) \leqq \sum_{i}\left(h_{i} \mid \phi\left(a_{i}\right) h_{i}\right) .
$$

Any element in the interior of $M_{2 s+}$ is a sum of two extreme elements of $M_{2 s+}$. Therefore we may assume that all $a_{i}$ are extreme (here "extreme" means: "belonging to an extreme ray"). Moreover we may remove from (5.7) all couples $\left(a_{i}, h_{i}\right)$ such that $a_{i}$ is proportional to $e^{\prime}$. This operation decreases the value of the RHS of (5.10) and does not affect the conditions (5.8) and (5.9). (At most it may change $k^{\prime}$, but $k^{\prime}$ does not enter (5.10).)

Extreme rays (except the one generated by $e^{\prime}$ ) are generated by element of the form $\left(\begin{array}{ll}1, & t \\ t, & t^{2}\end{array}\right)$, where $t \in \mathbb{R}$. Therefore

$$
a_{i}=\alpha_{i}\left(\begin{array}{ll}
1, & t_{i} \\
t_{i}, & t_{i}^{2}
\end{array}\right)
$$


where $\alpha_{i} \geqq 0, t_{i} \in \mathbb{R}$. Using (5.8) and (5.9) one gets immediately $\sum \alpha_{i} \leqq 1, \sum \alpha_{i} h_{i}=k$ and $\sum \alpha_{i} t_{i} h_{i}=0$. Now (5.10) follows immediately from (5.3).

Q.E.D.

Now, we may prove the main result of this section.

Theorem 5.3. Assume that (5.1) is nonextendible. Then $\phi$ is a Jordan representation:

$$
\phi\left(a^{2}\right)=\phi(a)^{2}
$$

for any $a \in M_{2 s}$.

Proof. Let $e, e^{\prime}$ be one-dimensional orthogonal projections in $M_{2 s}$ and $h \in H$. We put

$$
\alpha=e \otimes \phi\left(e^{\prime}\right) h+e^{\prime} \otimes\left(\phi\left(e^{\prime}\right) h-h\right) .
$$

By direct computation one checks that $\phi(\alpha)=0$. According to Proposition 2.2 (b) we have $\|\alpha\|_{\phi}=0$. Now using (5.2) we get

$$
\left(\phi\left(e^{\prime}\right) h \mid \phi(e) \phi\left(e^{\prime}\right) h\right)=0
$$

and

$$
\phi(e) \phi\left(e^{\prime}\right) h=0 .
$$

This way we proved that

$$
\phi(e) \phi\left(e^{\prime}\right)=0
$$

for any two one-dimensional orthogonal projections $e, e^{\prime} \in M_{2 s}$. We shall show that this property implies (5.11). At first we have

$$
\begin{gathered}
\phi(e)^{2}=\phi(e)\left(\phi(e)+\phi\left(e^{\prime}\right)\right)=\phi(e), \\
\phi\left(e^{\prime}\right)^{2}=\left(\phi(e)+\phi\left(e^{\prime}\right)\right) \phi\left(e^{\prime}\right)=\phi\left(e^{\prime}\right) .
\end{gathered}
$$

The Equations (5.12), (5.13), and (5.14) imply (5.11) for any $a$ of the form $\lambda e+\lambda^{\prime} e^{\prime}$, where $\lambda, \lambda^{\prime} \in \mathbb{R}$. On the other hand any selfadjoint element of $M_{2 s}$ can be written in this form ( $e$ and $e^{\prime}$ have to be chosen in a suitable way). Therefore (5.11) holds for any selfadjoint $a$.

To extend this formula for arbitrary $a$ we use the identity:

$$
(a+b i)^{2}=(1-i) a^{2}+i(a+b)^{2}-(1+i) b^{2} .
$$

Remark. Jordan representations of $M_{2 s}$ are nonextendible positive maps. The proof of Theorem 4.1 applies in this case as well.

The following theorem describes the structure of Jordan representations of $M_{2 s}$.

Theorem 5.4. Let (5.1) be a Jordan representation of $M_{2 s}$. Then

1. There exists a representation

$$
\phi_{1}: M_{2} \rightarrow B(H)
$$

such that

$$
\phi(a)=\phi_{1}(a)
$$

for all $a \in M_{2 s}$. 
2. The carrier Hilbert space $H$ can be written as the tensor product $H=\mathbb{C}^{2} \otimes K$ in such a way that

$$
\phi(a)=a \otimes I_{K} .
$$

Proof. It is known [10] that any Jordan homomorphism $\phi$ satisfies the following identity:

$$
\phi(a b c+c b a)=\phi(a) \phi(b) \phi(c)+\phi(c) \phi(b) \phi(a)
$$

for any $a, b, c$ belonging to the domain of $\phi$. In particular

$$
\phi(a b a)=\phi(a) \phi(b) \phi(a) \text {. }
$$

Let (5.15) be a linear mapping such that:

$$
\begin{aligned}
\phi_{1}\left(\begin{array}{ll}
1 & 0 \\
0 & 0
\end{array}\right) & =\phi\left(\begin{array}{ll}
1 & 0 \\
0 & 0
\end{array}\right), \phi_{1}\left(\begin{array}{ll}
0 & 1 \\
0 & 0
\end{array}\right)=\phi\left(\begin{array}{ll}
1 & 0 \\
0 & 0
\end{array}\right) \phi\left(\begin{array}{ll}
0 & 1 \\
1 & 0
\end{array}\right) \phi\left(\begin{array}{ll}
0 & 0 \\
0 & 1
\end{array}\right) \\
\phi_{1}\left(\begin{array}{ll}
0 & 0 \\
1 & 0
\end{array}\right) & =\phi\left(\begin{array}{ll}
0 & 0 \\
0 & 1
\end{array}\right) \phi\left(\begin{array}{ll}
0 & 1 \\
1 & 0
\end{array}\right) \phi\left(\begin{array}{ll}
1 & 0 \\
0 & 0
\end{array}\right) ; \phi_{1}\left(\begin{array}{ll}
0 & 0 \\
0 & 1
\end{array}\right)=\phi\left(\begin{array}{ll}
0 & 0 \\
0 & 1
\end{array}\right) .
\end{aligned}
$$

It follows easily from (5.17) that $\phi_{1}\left(\begin{array}{ll}0 & 1 \\ 1 & 0\end{array}\right)=\phi\left(\begin{array}{ll}0 & 1 \\ 1 & 0\end{array}\right)$ i.e. that (5.16) is satisfied. After simple, but not interesting calculations with the use of (5.18) one also checks that $(5.15)$ is multiplicative.

The second part of the theorem follows from the representation theory.

Now, collecting the results: Theorem 1.7, Theorem 5.3 and Theorem 5.4 we get

\section{Theorem 5.5. Let}

$$
\phi:\left(M_{2 s}, M_{2 s+}, 1\right) \rightarrow\left(B(H), B_{+}(H), I\right) .
$$

Then there exist a Hilbert space $K$ and an isometric embeding

$$
i: H \rightarrow \mathbb{C}^{2} \otimes K
$$

such that

$$
\phi(a)=i *\left(a \otimes I_{K}\right) i
$$

for all $a \in M_{2 s}$.

The similar result holds for non-normalized positive maps. In this case (5.19) is not an isometry, but a bounded linear mapping.

The positive maps of $M_{2 s}$ are closely related to the second order polynomials of one real variable with the values being positive operators. The one-to-one correspondence is given by the formula

$$
w_{2}(t)=\phi\left(\begin{array}{ll}
1, & t \\
t, & t^{2}
\end{array}\right)
$$


The Theorem 5.5 implies the following nice result:

Any second order polynomial $w_{2}(t)$ of one real variable $t$ with values in $B_{+}(H)$ can be factorized:

$$
w_{2}(t)=w_{1}(t)^{*} w_{1}(t)
$$

where $w_{1}(t)$ is a first order polynomial with values in $B\left(H, H_{1}\right) ; H_{1}$ is a Hilbert space.

Indeed if

$$
\begin{aligned}
H_{1} & =\mathbb{C}^{2} \otimes K \\
w_{1}(t) & =\left(\left(\begin{array}{ll}
1, & \\
0, & 0
\end{array}\right) \otimes I_{K}\right) i
\end{aligned}
$$

then (5.22) follows from (5.20) and (5.21).

Remark. It is interesting to consider factorizations of the type (5.22) with the smallest possible $H_{1}$. One can always assume that $H_{1}$ is generated by $\left\{w_{1}(t) h: t \in \mathbb{R}\right.$, $h \in H\}$. In this case $\operatorname{dim} H_{1} \leqq 2 \operatorname{dim} H$. By using differential geometry methods Michel improved this result and got $\operatorname{dim} H_{1}=\operatorname{dim} H$ [14].

\section{Nonextendible Positive Maps of the Matrix Algebra $M_{2}$}

In the previous section we showed that any nonextendible normalized positive map of $M_{2 s}$ is a Jordan representation. The situation changes rapidly when one replace $M_{2 s}$ by the full matrix algebra $M_{2}$. In [20] we proved that there exists a positive map $M_{2} \rightarrow M_{4}$ of non-jordanian type. By the procedure described in the Section 2 one may construct a nonextendible positive map $M_{2} \rightarrow B(H)$ not being Jordan representation. It is however very difficult to carry out such a construction in an explicite way.

In this section we construct a large class of nonextendible normalized positive maps of $M_{2}$ different from Jordan representations. This class does not contain all such maps, but we believe that the methods used in this section will give in the nearest future the fair classification of nonextendible positive maps of the matrix algebras.

Let $X$ be a two-dimensional Hilbert space. Elements of $M_{2}$ represent bounded operators acting on $X$. Thus, in what follows we shall deal with the operator algebra $B(X)$ instead of $M_{2}$.

We denote by $\bar{X}$ the Hilbert space complex conjugate to $X$. It means that $\bar{X}$ is a two-dimensional Hilbert space and that an antilinear, norm-preserving mapping

$$
X \ni x \rightarrow \bar{x} \in \bar{X}
$$

is given. We shall identify $B(X)$ with $\bar{X} \otimes X$ setting

$$
\mid x)(y \mid=\bar{y} \otimes x
$$

for any $x, y \in X$. The cone of positive elements $B_{+}(X)$ is generated by $\{\bar{x} \otimes x: x \in X\}$. 
The carrier Hilbert spaces of nonextendible maps constructed below concide with the symmetric tensor product of many copies of $X$.

Let $X^{k}$ denote the subspace of $X \otimes X \otimes \ldots \otimes X$ (the tensor product of $k$-copies of $X$ ) containing all symmetric tensors. $X^{k}$ carries the natural Hilbert space structure. One can check that $\operatorname{dim} X^{k}=k+1$.

$X^{k+1}$ can be considered as a subspace of $X^{k} \otimes X^{1}$. We denote by

$\pi: X^{k} \otimes X^{1} \rightarrow X^{k+1}$

the orthogonal projection (the symmetrization operator) and consequently by

$$
\pi^{*}: X^{k+1} \rightarrow X^{k} \otimes X^{1}
$$

the natural embeding.

The symmetric tensor product of two elements $p \in X^{k}$ and $q \in X^{1}$ will be denoted by $p q$ :

$$
p q=\pi(p \otimes q) \text {. }
$$

In particular $x^{k}$ denotes the (symmetric) tensor product of $k$-copies of $x \in X$. It is known that $\left\{x^{k}: x \in X\right\}$ generates linearly $X^{k}$.

Symmetric tensor algebra is an integral domain:

$$
(p q=0) \Leftrightarrow\left(\begin{array}{cr}
\text { either } p=0 \\
\text { or } & q=0
\end{array}\right)
$$

for any $p \in X^{k}$ and $q \in X^{1}$.

Remark. All vector spaces, we deal with in this section are finite-dimensional.

In the following theorems $H=X^{3}$ and $Q=X^{2}$. Theorem 6.1 gives examples of nonextendible positive maps $B(X) \rightarrow B(H)$ not being Jordan representation. Theorem 6.2 shows that the assumptions of Theorem 6.1 are selfconsistent. Theorem 6.3 shows that the nonextendible positive map constructed by the method described in Theorem 6.1 depends essentially on the "initial data" of this construction, so we have the continuum of essentially different (non equivalent) nonextendible positive maps.

Theorem 6.1. Let $\sigma: Q \rightarrow Q$ be a hermitian invertible operator. For any $p, q \in Q$, $x, y, r, s \in X$ we put

$$
\begin{aligned}
& (p \mid q)_{\sigma}=(p \mid \sigma q), \\
& (\bar{x} \otimes s \mid \bar{y} \otimes r)_{\hat{\sigma}^{-1}}=\left(y s \mid \sigma^{-1}(x r)\right) .
\end{aligned}
$$

Clearly these formulae introduce hermitian sesquilinear forms $\sigma$ and $\hat{\sigma}^{-1}$ defined on $Q$ and $\bar{X} \otimes X$. Assume that the signatures

$$
\begin{aligned}
& \operatorname{sign} \sigma=(+,+,-), \\
& \operatorname{sign} \hat{\sigma}^{-1}=(+,-,-,-) .
\end{aligned}
$$


Then

1. The operator $\varrho: X \otimes H \rightarrow X \otimes H$ introduced by the following diagram (the unnamed arrows represent the identity map $X \rightarrow X)$ :

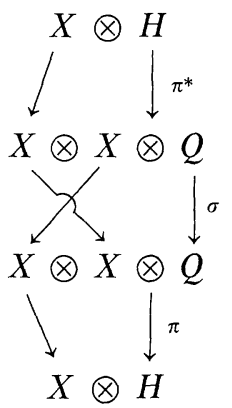

is invertible.

2. The linear map

$\phi: B(X) \rightarrow B(H)$

defined by the formula

$$
(k \mid \phi(\bar{x} \otimes y) h)=\left(x \otimes k \mid \varrho^{-1}(y \otimes h)\right)
$$

(where $x, y \in X ; k, h \in H$ ) is positive. In general (6.8) is not normalized, but in any case $R=\phi(1)$ is invertible. In what follows $\phi^{\prime}$ denotes the normalized positive map obtained by the normalization procedure:

$$
\phi^{\prime}(a)=R^{-1 / 2} \phi(a) R^{-1 / 2} .
$$

3. $\phi^{\prime}$ is nonextendible.

4. $\phi^{\prime}$ is irreducible.

5. $\phi^{\prime}$ is not Jordan representation.

Before the proof we remind the elementary facts of the "signature calculus" of hermitian sesquilinear forms.

Let $(\cdot \mid \cdot)_{\alpha}$ be a hermitian sesquilinear form defined on a complex vector space $K$. Then $K$ admits the decomposition

$$
K=K_{+} \oplus K_{0} \oplus K_{-}
$$

such that $\alpha$ is strictly positive on $K_{+}$(i.e. $(k \mid k)_{\alpha} \geqq 0$ for all $k \in K_{+}$and the equality holds only for $k=0$ ), $\alpha$ is strictly negative on $K_{-}$and $\alpha$ vanishes on $K_{0}$. Moreover $K_{+}, K_{0}$ and $K_{-}$are mutually $\alpha$-orthogonal.

It turns out that the dimensions of the spaces entering (6.9) are determined uniquely by $\alpha$ (Sylvester's law of inertia). The signature of $\alpha$ is by definition the collection of signs:

$$
\operatorname{sign} \alpha=(\underbrace{+++\ldots+}_{\begin{array}{c}
\operatorname{dim} K_{+} \\
\text {times }
\end{array}}, \underbrace{00 \ldots 0}_{\begin{array}{c}
\operatorname{dim} K_{0} \\
\text { times }
\end{array}}, \underbrace{-\ldots \ldots-}_{\begin{array}{c}
\operatorname{dim} K_{-} \\
\text {times }
\end{array}}) .
$$

If $s$ is a signature then we denote by $s_{+}, s_{0}$ and $s_{-}$the number of signs,+ 0 and - entering $s$. 
Example. Let $\gamma$ be the hermitian sesquilinear form on $X \otimes X$ such that

$$
\left(x^{\prime} \otimes y^{\prime} \mid x \otimes y\right)_{\gamma}=\left(x^{\prime} \mid y\right)\left(y^{\prime} \mid x\right)
$$

for all $x^{\prime}, y^{\prime}, x, y \in X$. Then

$$
\operatorname{sign} \gamma=(+++-) \text {. }
$$

Indeed $\gamma$ is strictly positive on the three-dimensional subspace of symmetric tensors and strictly negative on the one-dimensional subspace of skewsymmetric tensors.

Now, let $\alpha$ be a nondegenerate (i.e. $\operatorname{dim} K_{0}=0$ ) hermitian sesquilinear form defined on $K, K^{\prime}$ be a subspace of $K$ and $K^{\prime \prime}$ be the subspace containing all vectors $\alpha$-orthogonal to $K^{\prime}$. We shall call $K^{\prime \prime}$ the $\alpha$-orthocomplement of $K^{\prime}$. (Note that in general $K^{\prime}+K^{\prime \prime} \neq K$, however always $\operatorname{dim} K^{\prime}+\operatorname{dim} K^{\prime \prime}=\operatorname{dim} K$.) Let $\alpha^{\prime}$ and $\alpha^{\prime \prime}$ denote the restrictions of $\alpha$ to $K^{\prime}$ and $K^{\prime \prime}$ respectively. It turns out that $\operatorname{sign} \alpha^{\prime \prime}$ is determined uniquely by $\operatorname{sign} \alpha$ and $\operatorname{sign} \alpha^{\prime}$. We shall write

$$
\operatorname{sign} \alpha^{\prime \prime}=\operatorname{sign} \alpha \ominus \operatorname{sign} \alpha^{\prime} .
$$

The computation rule for this subtruction is rather simple ( $s$ and $s^{\prime}$ are signatures)

$$
\begin{aligned}
& \left(s \ominus s^{\prime}\right)_{+}=s_{+}-s_{+}^{\prime}-s_{0}^{\prime} \\
& \left(s \ominus s^{\prime}\right)_{0}=s_{0}^{\prime} \\
& \left(s \ominus s^{\prime}\right)_{-}=s_{-}-s_{-}^{\prime}-s_{0}^{\prime} .
\end{aligned}
$$

For example (we use the obvious short-hand notation)

$$
\begin{aligned}
& \left(+{ }^{6}-{ }^{2}\right) \ominus(++0-)=(+++0) \\
& \left(+{ }^{7}-{ }^{5}\right) \ominus(+---)=\left(+{ }^{6}-{ }^{2}\right) .
\end{aligned}
$$

Now, let $\alpha$ and $\beta$ be hermitian sesquilinear forms defined on complex vector spaces $K$ and $L$ respectively. The tensor product of these forms $\alpha \otimes \beta$ is a sesquilinear hermitian form defined on $K \otimes L$ :

$$
\left(k \otimes l \mid k^{\prime} \otimes l^{\prime}\right)_{\alpha \otimes \beta}=\left(k \mid k^{\prime}\right)_{\alpha}\left(l \mid l^{\prime}\right)_{\beta}
$$

for all $k, k^{\prime} \in K$ and $l, l^{\prime} \in L$. The signature of $\alpha \otimes \beta$ is determined uniquely by those of $\alpha$ and $\beta$. We shall write

$$
\operatorname{sign} \alpha \otimes \beta=\operatorname{sign} \alpha \otimes \operatorname{sign} \beta .
$$

To calculate the tensor product of two signatures $s$ and $s^{\prime}$ one has to multiply each sign from $s$ by each sign from $s^{\prime}$ according to the usual rule. The collection of obtained signs is $s \otimes s^{\prime}$. For example

$$
(+++-) \otimes(++-)=\left(+{ }^{7}-{ }^{5}\right) .
$$

Now, let $\alpha$ be a hermitian sesquilinear form on $K$ and $A: L \rightarrow K$ be a surjective linear map. One may introduce the inverse image $A^{-1} \alpha$. This is a hermitian sesquilinear form on $L$ such that

$$
\left(l \mid l^{\prime}\right)_{A^{-1} \alpha}=\left(A l \mid A l^{\prime}\right)_{\alpha}
$$


for all $l, l^{\prime} \in L$. Then the signature of $A^{-1} \alpha$ can be obtained from that of $\alpha$ by adding $\operatorname{dim}(\operatorname{ker} A)$ signs 0 . We shall write

$$
\operatorname{sign}\left(A^{-1} \alpha\right)=\operatorname{sign} \alpha \oplus\left(0^{\operatorname{dim}(\operatorname{ker} A)}\right) .
$$

In particular, if $A$ is one-to-one map then $\operatorname{sign}\left(A^{-1} \alpha\right)=\operatorname{sign} \alpha$.

We shall use in the following proof all rules of the signature calculus mentioned above.

Proof of Theorem 6.1. Ad 1. We introduce the sesquilinear hermitian form $\varrho$ defined on $X \otimes H$ setting for all $h, k \in H$ and $x, y \in X$ :

$(x \otimes h \mid y \otimes k)_{\varrho}=(x \otimes h \mid \varrho(y \otimes k))$.

We shall show, that

$\operatorname{sign} \varrho=\left(+{ }^{6}-{ }^{2}\right)$.

In particular this means that the form $\varrho$ is not degenerate and the invertibility of the operator $\varrho$ follows immediately.

According to our notation $H \subset X \otimes Q$ and $X \otimes H \subset X \otimes X \otimes Q$. In $X \otimes X \otimes Q$ we introduce another form $\varrho$ setting for all $x, y, x^{\prime}, y^{\prime} \in X$ and $q, q^{\prime} \in Q$ :

$\left(x \otimes y \otimes q \mid x^{\prime} \otimes y^{\prime} \otimes q^{\prime}\right)_{\tilde{\varrho}}=\left(x \mid y^{\prime}\right)\left(y \mid x^{\prime}\right)\left(q \mid q^{\prime}\right)_{\sigma}$.

Let us notice that the form $\varrho$ coincides with the restriction of $\tilde{\varrho}$ to $X \otimes H$. This fact follows immediately from the Definitions (6.17) and (6.19) and the Diagram (6.6).

The signature of $\varrho$ can be easily calculated. Indeed $\tilde{\varrho}=\gamma \otimes \sigma$, where $\gamma$ is the form on $X \otimes X$ introduced by (6.10). Therefore [cf. (6.11), (6.4), and (6.15)]

$$
\begin{aligned}
\operatorname{sign} \tilde{\varrho} & =(+++-) \otimes(++-) \\
& =\left(+{ }^{7}-{ }^{5}\right) .
\end{aligned}
$$

Let $L$ be the $\tilde{\varrho}$-orthocomplement of $X \otimes H$ and let $\varrho^{\prime}$ denote the restriction of $\tilde{\varrho}$ to $L$. We have $\operatorname{dim} L=\operatorname{dim} X \otimes X \otimes Q-\operatorname{dim} X \otimes H=2 \cdot 2 \cdot 3-2 \cdot 4=4$. It turns out that

$$
\operatorname{sign} \varrho^{\prime}=\operatorname{sign} \hat{\sigma}^{-1} \text {. }
$$

Now, according to (6.12) we have

$$
\begin{aligned}
\operatorname{sign} \varrho & =\operatorname{sign} \tilde{\varrho} \ominus \operatorname{sign} \varrho^{\prime} \\
& =\left(++^{7}-{ }^{5}\right) \ominus(+---)
\end{aligned}
$$

and (6.18) follows [cf. (6.14)].

To end this part of the proof we have to show (6.20). Let $\left(e_{1}, e_{2}\right)$ be an orthonormal basis of $X$. For any $x \in X$ we put

$$
j(x)=\left(x \mid e_{1}\right) e_{2}-\left(x \mid e_{2}\right) e_{1} .
$$

It can be checked immediately that

$$
j: X \rightarrow X
$$


is an antilinear, norm-preserving mapping and that

$$
(x \mid j(x))=0
$$

for any $x \in X$. Moreover

$$
j^{2}=-I \text {. }
$$

Now, let us introduce a linear mapping

$$
\varphi: \bar{X} \otimes X \rightarrow X \otimes X \otimes Q
$$

setting for all $x, y \in X$ :

$$
\varphi(\bar{x} \otimes y)=e_{1} \otimes j(x) \otimes \sigma^{-1}\left(y e_{2}\right)-e_{2} \otimes j(x) \otimes \sigma^{-1}\left(y e_{1}\right) .
$$

Using this definition and formulae (6.19) and (6.21) we get after simple calculations

$$
\left(z^{\prime} \otimes z \otimes q \mid \varphi(\bar{x} \otimes y)\right)_{\varrho}=\left(z^{\prime} \mid j(x)\right)(q \mid y j(z))
$$

for any $z, z^{\prime}, x, y \in X$ and $q \in Q$. If $q=z^{2}$ then, in virtue of (6.23), the second factor on the RHS of the above formula vanishes and we have

$$
\left(z^{\prime} \otimes z^{3} \mid \varphi(\bar{x} \otimes y)\right)_{\tilde{Q}}=0 .
$$

This equation shows that $\varphi(\bar{x} \otimes y)$ is $\tilde{\varrho}$-orthogonal to $X \otimes H$. Therefore $\varphi(\bar{X} \otimes X) C L$. If $\varphi$ is invertible (i.e. $\operatorname{ker} \varphi=\{0\}$ ), then $\operatorname{dim} \varphi(\bar{X} \otimes X)=\operatorname{dim} \bar{X} \otimes X=$ $4=\operatorname{dim} L$, and we get

$$
\varphi(\bar{X} \otimes X)=L .
$$

Let $x, y, x^{\prime}, y^{\prime} \in X$. Making use of (6.25), (6.26), (6.21), (6.24), and (6.3) we have

$$
\begin{aligned}
& \left(\varphi\left(\bar{x}^{\prime} \otimes y^{\prime}\right) \mid \varphi(\bar{x} \otimes y)\right)_{\tilde{\varrho}} \\
& \quad=\left(e_{1} \otimes j\left(x^{\prime}\right) \otimes \sigma^{-1}\left(y^{\prime} e_{2}\right)-e_{2} \otimes j\left(x^{\prime}\right) \otimes \sigma^{-1}\left(y^{\prime} e_{1}\right) \mid \varphi(\bar{x} \otimes y)\right)_{\tilde{Q}} \\
& \quad=\left(e_{1} \mid j(x)\right)\left(\sigma^{-1}\left(y^{\prime} e_{2}\right) \mid y j^{2}\left(x^{\prime}\right)\right)-\left(e_{2} \mid j(x)\right)\left(\sigma^{-1}\left(y^{\prime} e_{1}\right) \mid y j^{2}\left(x^{\prime}\right)\right) \\
& \quad=\left(\sigma^{-1}\left(y^{\prime} j^{2}(x)\right) \mid y j^{2}\left(x^{\prime}\right)\right)=\left(y^{\prime} x \mid \sigma^{-1}\left(y x^{\prime}\right)\right)=\left(\bar{x}^{\prime} \otimes y^{\prime} \mid \bar{x} \otimes y\right)_{\hat{\sigma}^{-1}} .
\end{aligned}
$$

Therefore, for any $a, b \in \bar{X} \otimes X$ :

$$
(\varphi(a) \mid \varphi(b))_{\tilde{Q}}=(a \mid b)_{\hat{\sigma}^{-1}} .
$$

Since $\hat{\sigma}^{-1}$ is not degenerate [cf. (6.5)], $\varphi$ is invertible and (6.27) is justified. Now, (6.20) follows directly from (6.27) and (6.28). This ends the first part of the proof.

Ad 2. Let $x \in X$ and $x \neq 0$. The sesquilinear hermitian form corresponding to the operator $\phi(\bar{x} \otimes x)$ will be denoted by the same symbol:

$$
(k \mid h)_{\phi(\bar{x} \otimes x)}=(k \mid \phi(\bar{x} \otimes x) h)
$$

for all $h, k \in H$.

It is sufficient to show that this form is nonnegative. We shall prove more:

$$
\operatorname{sign} \phi(\bar{x} \otimes x)=(+++0) \text {. }
$$


For any $h, k \in H$ we have

$$
\begin{aligned}
(k \mid h)_{\phi(\bar{x} \otimes x)} & =(k \mid \phi(\bar{x} \otimes x) h)=\left(x \otimes k \mid \varrho^{-1}(x \otimes h)\right) \\
& =\left(\varrho^{-1}(x \otimes k) \mid \varrho^{-1}(x \otimes h)\right)_{\varrho} .
\end{aligned}
$$

Therefore sign $\phi(\bar{x} \otimes x)$ coincides with the signature of the form $\varrho$ restricted to $\varrho^{-1}(x \otimes H)$. One can easily check that $\varrho$-orthocomplement of $\varrho^{-1}(x \otimes H)$ equals to $x^{\prime} \otimes H$, where $x^{\prime} \neq 0$ is an element of $X$ orthogonal to $x$ [one may take $x^{\prime}=j(x)$, cf. (6.23)]. Therefore

$$
\operatorname{sign} \phi(\bar{x} \otimes x)=\operatorname{sign} \varrho \ominus \operatorname{sign} \varrho_{x^{\prime}}
$$

where $\varrho_{x^{\prime}}$ denotes the restriction of $\varrho$ to $x^{\prime} \otimes H$.

For any $y \in X, A_{\bar{y}}$ will denote the scalar multiplication of elements of $X \otimes Q$ by $y$. More precisely $A_{\bar{y}}$ is a linear operator $X \otimes Q \rightarrow Q$ such that

$$
A_{\bar{y}}(x \otimes q)=(y \mid x) q .
$$

Now, the formula (6.19) can be written in another form

$$
\left(x \otimes y \otimes q \mid x^{\prime} \otimes y^{\prime} \otimes q^{\prime}\right)_{\tilde{Q}}=\left(A_{\bar{x}^{\prime}}(y \otimes q) \mid A_{\bar{x}}\left(y^{\prime} \otimes q^{\prime}\right)\right)_{\sigma} .
$$

In particular

$$
\left(x^{\prime} \otimes k \mid x^{\prime} \otimes h\right)_{\varrho}=\left(A_{\bar{x}^{\prime}} \mid A_{\bar{x}^{\prime}} h\right)_{\sigma}
$$

for any $k, h \in H$. This shows that $\varrho_{x^{\prime}}$ coincides with the inverse image of $\sigma$. One can check that the mapping

$$
A_{\bar{x}^{\prime}}: H \rightarrow Q
$$

is surjective. Taking into account the fact that $\operatorname{dim} H=\operatorname{dim} Q+1$ we obtain [cf. (6.16)]

$$
\begin{aligned}
\operatorname{sign} \varrho_{x^{\prime}} & =\operatorname{sign} \sigma \oplus(0) \\
& =(++0-) .
\end{aligned}
$$

Now (6.29) follows immediately from (6.30) and (6.18) [cf. (6.13)].

Ad 3. Let $j$ be the antilinear, norm-preserving operator acting on $X$ introduced by (6.21). For any $x \in X$ we put

$$
k_{x}=\pi\left(j(x) \otimes \sigma\left(x^{2}\right)\right) .
$$

We have [cf. diagram (6.6)]

$$
\varrho\left(j(x) \otimes x^{3}\right)=x \otimes \pi\left(j(x) \otimes \sigma\left(x^{2}\right)\right) .
$$

Therefore

$$
\varrho^{-1}\left(x \otimes k_{x}\right)=j(x) \otimes x^{3}
$$

and for any $h \in H$ :

$$
\begin{aligned}
\left(h \mid \phi(\bar{x} \otimes x) k_{x}\right) & =\left(x \otimes h \mid \varrho^{-1}\left(x \otimes k_{x}\right)\right) \\
& =\left(x \otimes h \mid j(x) \otimes x^{3}\right)=0
\end{aligned}
$$


on the basis of (6.23). It shows that

$$
\phi(\bar{x} \otimes x) k_{x}=0 \text {. }
$$

Let us note that this result is in full coherence with (6.29); we found the vector responsible for 0 in the signature (6.29). (6.29) implies that $k_{x}$ is the only vector satisfying (6.33). It is obvious that $k_{x}$ is a nontrivial function of $x$ : there exist at least two elements $x, y X$ such that $k_{x}$ and $k_{y}$ are not proportional. Then $\phi(\bar{x} \otimes x+\bar{y} \otimes y)$ is strictly positive i.e. invertible. The invertibility of $R=\phi(1)$ follows immediately.

Now let

$$
k_{x}^{\prime}=R^{1 / 2} k_{x} .
$$

Then

$$
\phi^{\prime}(\bar{x} \otimes x) k_{x}^{\prime}=0 .
$$

Now, let us consider the subspace of $B(X) \otimes H=\bar{X} \otimes X \otimes H$ generated by all elements of the form $\bar{x} \otimes x \otimes k_{x}^{\prime}$. We denote this subspace by $N$. According to Theorem 3.3 it is sufficient to show that

$$
\operatorname{dim} N=\operatorname{dim} H(\operatorname{dim} B(X)-1)=4 \cdot 3=12 .
$$

It will be easier to calculate the dimension of an isomorphic image of $N$. Let $W: \bar{X} \otimes X \otimes H \rightarrow \bar{X} \otimes X \otimes H$ be an invertible operator introduced by the diagram

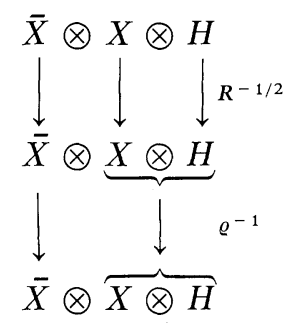

In virtue of (6.32) we have

$$
W\left(\bar{x} \otimes x \otimes k_{x}^{\prime}\right)=\bar{x} \otimes j(x) \otimes x^{3} .
$$

Therefore $W(N)$ is generated by all vectors of the form

$$
\bar{x} \otimes j(x) \otimes x^{3} \text {. }
$$

Like in the theory of holomorphic functions, we may consider $x$ and $\bar{x}$ as independent variables. It means [remember that $j(x)$ depends on $x$ antilinearly] that $W(N)$ is generated by all vectors of the form

$$
\bar{y} \otimes j(y) \otimes x^{3}
$$

where $x, y \in X$. Therefore

$$
W(N)=S \otimes H
$$

where $S$ is a subspace of $\bar{X} \otimes X$ generated by all vectors of the form $\bar{y} \otimes j(y)$. 
Let us consider the linear map of $\bar{X} \otimes \bar{X}$ onto $\bar{X} \otimes X$ sending $\bar{y} \otimes \bar{x}$ onto $\bar{y} \otimes j(x)$. Clearly this is an invertible map and $S$ coincides with the image of $\bar{X}^{2} C \bar{X} \otimes \bar{X}$. Therefore $\operatorname{dim} S=\operatorname{dim} \bar{X}^{2}=3$ and (6.34) follows.

Ad 4. Let $P$ be a projection operator acting on $H$ and commuting with all $\varphi^{\prime}(\bar{x} \otimes x)$. We know that $h=k_{x}^{\prime}$ is the only vector satisfying equation $\phi^{\prime}(\bar{x} \otimes x) h=0$. On the other hand one easily checks that $h=P k_{x}^{\prime}$ satisfies this equation as well. Therefore $P k_{x}^{\prime}$ is either $k_{x}^{\prime}$ or 0 and we have either $P=I$ or $P=0$ (it is known that $\left\{k_{x}: x \in X\right\}$ generates $H$, cf. the remark at the end of the Section 3).

Ad 5. We know, that carrier Hilbert spaces of irreducible Jordan representations of $M_{2}$ are two-dimensional.

Q.E.D.

Remark. In our signature calculations we took into account particular values of $\operatorname{sign} \sigma$ and $\operatorname{sign} \hat{\sigma}^{-1}$ given by (6.4) and (6.5). To be more general one may calculate sign $\phi(\bar{x} \otimes x)$ without using these data. The result is

$\operatorname{sign} \phi(\bar{x} \otimes x)=(++-) \otimes \operatorname{sign} \sigma \ominus \operatorname{sign} \hat{\sigma}^{-1} \ominus(0)$.

Let us note that we get (6.29) assuming that

$$
\begin{aligned}
\operatorname{sign} \sigma & =(+++) \\
\operatorname{sign} \hat{\sigma}^{-1} & =(++--) .
\end{aligned}
$$

It turns out however that (6.36) can not be realized by any operator $\sigma: Q \rightarrow Q$. The same can be said about the other possibility:

$$
\begin{aligned}
\operatorname{sign} \sigma & =(+--) \\
\operatorname{sign} \hat{\sigma}^{-1} & =(----)
\end{aligned}
$$

also leading to (6.29). On the contrary, the set of operators $\sigma$ satisfying (6.4) and (6.5) is not empty:

Theorem 6.2. Let $\sigma: Q \rightarrow Q$ be an invertible hermitian operator. Then the following two statements are equivalent:

I. $\sigma$ satisfies (6.4) and (6.5),

II. There exists a (not orthonormal in general) basis $(x, y)$ in $X$ such that:

$$
\begin{aligned}
\left(x^{2} \mid \sigma^{-1}\left(x^{2}\right)\right) & =\lambda_{1}+\lambda_{2}, \quad\left(x^{2} \mid \sigma^{-1}(x y)\right)=0, \quad\left(x^{2} \mid \sigma^{-1}\left(y^{2}\right)\right)=\lambda_{1}-\lambda_{2}, \\
\left(x y \mid \sigma^{-1}\left(x^{2}\right)\right) & =0, \quad\left(x y \mid \sigma^{-1}(x y)\right)=\lambda_{3}, \quad\left(x y \mid \sigma^{-1}\left(y^{2}\right)\right)=0, \\
\left(y^{2} \mid \sigma^{-1}\left(x^{2}\right)\right) & =\lambda_{1}-\lambda_{2}, \quad\left(y^{2} \mid \sigma^{-1}(x y)\right)=0, \quad\left(y^{2} \mid \sigma^{-1}\left(y^{2}\right)\right)=\lambda_{1}+\lambda_{2}
\end{aligned}
$$

where $\lambda_{1}, \lambda_{2}-0$ and $\lambda_{1}+\lambda_{2}+\lambda_{3}<0$.

Proof. We shall only prove that $\mathrm{II} \Rightarrow \mathrm{I}$. From our point of view this is more important part of Theorem 6.2. It shows that Theorem 6.1 really gives us examples of nonextendible positive maps. The proof that $\mathrm{I} \Rightarrow \mathrm{II}$ is not difficult but rather long and will be omitted.

One may summarize (6.37) saying that

$$
\left(\begin{array}{ccc}
\lambda_{1}+\lambda_{2}, & 0, & \lambda_{1}-\lambda_{2} \\
0, & \lambda_{3}, & 0 \\
\lambda_{1}-\lambda_{2}, & 0, & \lambda_{1}+\lambda_{2}
\end{array}\right)
$$


is the matrix representing the form $\sigma^{-1}$ in the basis $\left(x^{2}, x y, y^{2}\right)$. The eigenvalues of this matrix can be easily calculated. One obtains $2 \lambda_{1}, 2 \lambda_{2}, \lambda_{3}$. Therefore $\operatorname{sign} \sigma^{-1}=(++-)\left(\lambda_{3}\right.$ is negative) and (6.4) is verified (the signatures of $\sigma$ and $\sigma^{-1}$ always coincide).

Using the Definition (6.3) one can compute the matrix representing the form $\hat{\sigma}^{-1}$ in the basis $(\bar{x} \otimes x, \bar{x} \otimes y, \bar{y} \otimes x, \bar{y} \otimes y)$. The result is:

$$
\left(\begin{array}{cccc}
\lambda_{1}+\lambda_{2}, & 0, & 0, & \lambda_{3} \\
0, & \lambda_{3}, & \lambda_{1}-\lambda_{2}, & 0 \\
0, & \lambda_{1}-\lambda_{2}, & \lambda_{3}, & 0 \\
\lambda_{3}, & 0, & 0, & \lambda_{1}+\lambda_{2}
\end{array}\right) .
$$

The eigenvalues of this matrix equal to $\lambda_{1}+\lambda_{2}+\lambda_{3}, \lambda_{1}+\lambda_{2}-\lambda_{3}, \lambda_{1}-\lambda_{2}+\lambda_{3}$ and $-\lambda_{1}+\lambda_{2}+\lambda_{3}$. Only the second eigenvalue is positive. Therefore

$$
\operatorname{sign} \hat{\sigma}^{-1}=(+---) \text {. }
$$

It turns out that different operators $\sigma$ give rise to the essentially different positive nonextendible maps.

Theorem 6.3. Let $\phi$ and $\phi_{1}$ be two positive maps of $B(X)$ constructed according to Theorem 6.1. Assume that there exists an invertible operator $V \in B(H)$ such that $\phi(a)=V^{*} \phi_{1}(a) V$ for all $a \in B(X)$. Then the operators $\sigma$ and $\sigma_{1}$ corresponding to $\phi$ and $\phi_{1}$ respectively are proportional.

Proof. We have [cf. (6.31) and (6.33)]

$$
\begin{aligned}
\phi(\bar{x} \otimes x) \pi\left(j(x) \otimes \sigma\left(x^{2}\right)\right) & =0, \\
\phi_{1}(\bar{x} \otimes x) \pi\left(j(x) \otimes \sigma_{1}\left(x^{2}\right)\right) & =0 .
\end{aligned}
$$

Therefore $V \pi\left(j(x) \otimes \sigma\left(x^{2}\right)\right)$ must be proportional to $\pi\left(j(x) \otimes \sigma_{1}\left(x^{2}\right)\right)$. Denoting the coefficient by $\lambda_{x}$ we get

$$
V \pi\left(j(x) \otimes \sigma\left(x^{2}\right)\right)=\lambda_{x} \pi\left(j(x) \otimes \sigma_{1}\left(x^{2}\right)\right) .
$$

Treating again $\bar{x}$ and $x$ as independent variables we get

$$
V \pi\left(j(y) \otimes \sigma\left(x^{2}\right)\right)=\lambda_{x y} \pi\left(j(y) \otimes \sigma_{1}\left(x^{2}\right)\right)
$$

for all $x, y \in X$.

Let $x$ runs over $X$. Then $\pi\left(j(y) \otimes \sigma\left(x^{2}\right)\right)$ and $\pi\left(j(y) \otimes \sigma_{1}\left(x^{2}\right)\right)$ span the three dimesional subspace of $H$ orthogonal to $y^{3}$. The formula (6.39) tells that this subspace is $V$-invariant. Therefore $y^{3}$ is an eigenvector of $V^{*}$ and this fact holds for any $y \in X$. It follows easily that $V=\lambda I$, where $\lambda \in \mathbb{C}$.

Now, (6.38) can be simplified:

$$
\pi\left(j(x) \otimes\left[\lambda \sigma\left(x^{2}\right)-\lambda_{x} \sigma_{1}\left(x^{2}\right)\right]\right)=0 .
$$

We know that the kernel of the projection $\pi: X \otimes Q \rightarrow H$ does not contain any non-zero element of the form $y \otimes q$ [cf. (6.1)]. Therefore, using (6.40) we get

$$
\lambda \sigma\left(x^{2}\right)=\lambda_{x} \sigma_{1}\left(x^{2}\right) .
$$


It means that $x^{2}$ is an eigenvalue of $\sigma^{-1}{ }^{\circ} \sigma_{1}$. Then $\sigma^{-1}{ }_{\circ} \sigma_{1}$ must be proportional to $I_{Q}$ (the eigenvectors of other operators do not form a continuum generating the whole space).

Q.E.D.

It is not difficult to produce examples of irreducible nonextendible maps of $B(X)$ with higher dimensional carrier Hilbert spaces. To this end one generalizes Theorem 6.1 setting $H=X^{n}$ and $Q=X^{n-1}$. In this case $\hat{\sigma}^{-1}$ is defined on $\bar{X} \otimes X^{n-2}$. The formula (6.35) holds in this general case as well. In order to obtain

$$
\operatorname{sign} \phi(\bar{x} \otimes x)=\left(+{ }^{n} 0\right)
$$

we have to assume that the signatures $s=\operatorname{sign} \sigma$ and $s^{\prime}=\operatorname{sign} \hat{\sigma}^{-1}$ satisfy the following conditions:

$$
\begin{aligned}
s_{+}+2 s_{-} & =s_{-}^{\prime}+1 \\
s_{0}=s_{0}^{\prime} & =0 .
\end{aligned}
$$

The Theorem 6.3 is also valid in this general setting. On the contrary Theorem 6.2 strongly depends on the assumption $n=3$. We do not even know whether for given $s$ and $s^{\prime}$ satisfying (6.41) there exists an operator $\sigma$ such that $\operatorname{sign} \sigma=s$ and $\hat{\sigma}^{-1}=s^{\prime}$. It seems that the answer is negative in most cases.

In the end of this paper we would like to present the first example of a nonextendible positive map $\phi: M_{2} \rightarrow M_{4}$ found rather accidentally after long and unsuccesful attemps to construct such a map in a systematic way:

$$
\phi\left(\begin{array}{ll}
a, & b \\
c, & d
\end{array}\right)=\left(\begin{array}{cccc}
4 a-2 b-2 c+4 d, & -2 a+2 c, & 0, & 0 \\
-2 a+2 b, & 2 a, & c, & 0 \\
0, & b, & 2 d, & -2 c-d \\
0, & 0, & -2 b-d, & 4 a+2 d
\end{array}\right) .
$$

All the theory presented in this section is the result of the detail examination of this single numerical example.

Acknowledgement. This research was carried out in part at the author's home institution, where it was supported by the Polish Academy of Sciences (P.A.N.). It was completed during visits to Centre de Physique Theorique CNRS, Marseille and to Zentrum für Interdisziplinäre Forschung at the University of Bielefeld. The author would like to thank Professor K. Maurin for his interest and encouragement. He also thanks Professors R. Stora and L. Streit for their kind hospitality in Marseille and in Bielefeld.

The author is grateful to Professor H. Araki for pointing out an error in the previous version of this paper and to Professor D. Kastler for a remark leading to Theorem 4.7.

\section{References}

1. Averson, W. B.: Subalgebras of $C^{*}$-algebras. I. Acta Math. 123, 141-224(1969); II. 128, 271-308 (1972)

2. Calderón, A.P.: A note on biquadratic forms. Linear Algebra Appl. 7, 175-177 (1973)

3. Choi, M.-D.: Completely positive linear maps on complex matrices. Linear Algebra Appl. (to appear)

4. Choi, M.-D.: Positive semi-definite biquadratic forms (preprint)

5. Day, M. M.: Normed linear spaces. Berlin-Heidelberg-New York: Springer 1973 
6. Gorini,V., Kossakowski,A., Sudarshan,E.C.G.: Completely positive dynamical semigroups of $N$-level systems. The University of Texas at Austin, Preprint ORO 3992-200

7. Halmos, P. R.: Naive set theory. Princeton-London-Toronto: Van Nostrand Reinhold Company 1960

8. Ingarden, S. R., Woronowicz, S. L.: On mixed and pure observables. Institute of Physics, Nicholas Copernikus University Toruń, Preprint No. 297 (1975)

9. Jakubovic, V.A.: Factorization of symmetric matrix polynomials. Soviet Math. Dokt. 11, $1261-1264(1970)$

10. Kadison, R.V.: Isometries of operator algebras. Ann. Math. 54, 325 (1951)

11. Koga,T.: Synthesis of finite passive $n$-ports with prescribed positive real matrices of several variables IEEE Transactions on Circuit Theory. CT 15, 2 (1968)

12. Lindblad, G.: On the generators of quantum dynamical semigroups. Royal Institute of Technology, Department of Theoretical Physics, Stockholm, preprint TRITA-TFY-75-1

13. Maurin, K.: Methods of Hilbert space, PWN, Warszawa 1967

14. Michel, L.: private communication

15. Mielnik, B.: Generalized quantum mechanics. Commun. math. Phys. 37, 221-256 (1974)

16. Phelps, R.R.: Lectures on Choquet's theory. Princeton: Van Nostrand 1966

17. Rosenblum, M., Rovnyak, J.: The factorization problem for non-negative operator valued functions. Bull. Amer. Math. Soc. 77, 287-318 (1971)

18. Størmer, E.: Positive linear maps of $C^{*}$-algebras. Lecture Notes in Physics 29, 85--106. BerlinHeidelberg-New York: Springer 1974

19. Woronowicz, S. L.: Selfpolar forms and their applications to the $C^{*}$-algebra theory. Rep. Math. Phys. 6, 487-495 (1974)

20. Woronowicz, S.L.: Positive maps of low dimensional matrix algebras. Rep. Math. Phys. 10, $165-183(1976)$

Communicated by H. Araki

Received April 2, 1976 